\title{
The 1-Fixed-Endpoint Path Cover Problem is Polynomial on Interval Graphs
}

\author{
Katerina Asdre • Stavros D. Nikolopoulos
}

Received: 1 April 2008 / Accepted: 11 February 2009

(C) Springer Science+Business Media, LLC 2009

\begin{abstract}
We consider a variant of the path cover problem, namely, the $k$-fixedendpoint path cover problem, or $k \mathrm{PC}$ for short, on interval graphs. Given a graph $G$ and a subset $\mathcal{T}$ of $k$ vertices of $V(G)$, a $k$-fixed-endpoint path cover of $G$ with respect to $\mathcal{T}$ is a set of vertex-disjoint paths $\mathcal{P}$ that covers the vertices of $G$ such that the $k$ vertices of $\mathcal{T}$ are all endpoints of the paths in $\mathcal{P}$. The $k \mathrm{PC}$ problem is to find a $k$-fixed-endpoint path cover of $G$ of minimum cardinality; note that, if $\mathcal{T}$ is empty the stated problem coincides with the classical path cover problem. In this paper, we study the 1-fixed-endpoint path cover problem on interval graphs, or 1PC for short, generalizing the 1HP problem which has been proved to be NP-complete even for small classes of graphs. Motivated by a work of Damaschke (Discrete Math. 112:4964, 1993), where he left both 1HP and 2HP problems open for the class of interval graphs, we show that the 1PC problem can be solved in polynomial time on the class of interval graphs. We propose a polynomial-time algorithm for the problem, which also enables us to solve the 1HP problem on interval graphs within the same time and space complexity.
\end{abstract}

Keywords Perfect graphs · Interval graphs · Path cover · Fixed-endpoint path cover $\cdot$ Linear-time algorithms

\section{Introduction}

Framework-Motivation A well studied problem with numerous practical applications in graph theory is to find a minimum number of vertex-disjoint paths of a

K. Asdre · S.D. Nikolopoulos ( $₫)$

Department of Computer Science, University of Ioannina, P.O. Box 1186, 45110 Ioannina, Greece e-mail: stavros@cs.uoi.gr

K. Asdre

e-mail: katerina@cs.uoi.gr 
graph $G$ that cover the vertices of $G$. This problem, also known as the path cover problem (PC), finds application in the fields of database design, networks, code optimization among many others (see [1, 2, 19, 28]); it is well known that the path cover problem and many of its variants are NP-complete in general graphs [10]. A graph that admits a path cover of size one is referred to as Hamiltonian. Thus, the path cover problem is at least as hard as the Hamiltonian path problem (HP), that is, the problem of deciding whether a graph is Hamiltonian. The path cover problem is known to be NP-complete even when the input is restricted to several interesting special classes of graphs; for example, it is NP-complete on planar graphs [11], bipartite graphs [12], chordal graphs [12], chordal bipartite graphs [20] and strongly chordal graphs [20]. Bertossi and Bonuccelli [6] proved that the Hamiltonian Circuit problem is NP-complete on several interesting classes of intersection graphs.

Several variants of the HP problem are also of great interest, among which is the problem of deciding whether a graph admits a Hamiltonian path between two points (2HP). The $2 \mathrm{HP}$ problem is the same as the HP problem except that in $2 \mathrm{HP}$ two vertices of the input graph $G$ are specified, say, $u$ and $v$, and we are asked whether $G$ contains a Hamiltonian path beginning with $u$ and ending with $v$. Similarly, the 1HP problem is to determine whether a graph $G$ admits a Hamiltonian path starting from a specific vertex $u$ of $G$, and to find one if such a path does exist. Both $1 \mathrm{HP}$ and 2HP problems are also NP-complete in general graphs [10].

The path cover problem as well as several variants of it have been extensively studied due to their wide applicability in many fields. Some of these problems, of both theoretical and practical importance, are in the context of communication and/or transposition networks [29]. In such problems, we are given a graph (network) $G$ and two disjoint subsets $\mathcal{T}_{1}$ and $\mathcal{T}_{2}$ of vertices of $G$, and the objective is to determine whether $G$ admits $\lambda$ vertex-disjoint paths with several conditions on their endpoints with respect to $\mathcal{T}_{1}$ and $\mathcal{T}_{2}$, e.g., paths with both their endpoints in $\mathcal{T}_{1} \cup \mathcal{T}_{2}$, paths with one endpoint in $\mathcal{T}_{1}$ and the other in $\mathcal{T}_{2}$, etc. [3, 4, 29]; note that, the endpoints of a path $P$ are the first vertex and the last vertex visited by $P$.

A similar problem that has received increased attention in recent years is in the context of communication networks. The only efficient way to transmit high volume communication, such as in multimedia applications, is through disjoint paths that are dedicated to pairs of processors. To efficiently utilize the network, one needs a simple algorithm that, with minimum overhead, constructs a large number of edge-disjoint paths between pairs of two given sets $\mathcal{T}_{1}$ and $\mathcal{T}_{2}$ of requests.

Furthermore, in the study of interconnection networks, the reliability of the interconnection network subject to node failures corresponds to the connectivity of an interconnection graph. It is well-known that the connectivity of a graph $G$ is characterized in terms of vertex-disjoint paths joining a pair of vertices in $G$. Thus, one-to-many vertex-disjoint paths joining a vertex $s$ (source) and $k$ distinct vertices $t_{1}, t_{2}, \ldots, t_{k}$ (sinks) are required. A related work was presented by Park in [23].

Another related problem is the disjoint paths (DP) problem, which is defined as follows: Given a graph $G$ and pairs $\left(s_{1}, t_{1}\right),\left(s_{2}, t_{2}\right), \ldots,\left(s_{k}, t_{k}\right)$ of vertices of $G$, the objective is to determine whether $G$ admits $k$ vertex-disjoint paths $P_{1}, P_{2}, \ldots, P_{k}$ in $G$ such that $P_{i}$ joins $s_{i}$ and $t_{i}(1 \leq i \leq k)$. The problem was shown to be NP-complete by Karp [17] if $k$ is a variable part of the input. For fixed $k$, however, the problem 
is more tractable; a polynomial-time algorithm was described by Robertson and Seymour [25]. Note that, for $k=2$, there are several polynomial-time algorithms in the literature for the DP problem [26, 27, 30]. In contrast, the corresponding question for directed graphs $G$ where we seek directed paths $P_{1}, P_{2}, \ldots, P_{k}$ is NP-complete even for $k=2$ [9].

In [8], Damaschke provided a foundation for obtaining polynomial-time algorithms for several problems concerning paths in interval graphs, such as finding Hamiltonian paths and circuits, and partitions into paths. In the same paper, he stated that the complexity status of both $1 \mathrm{HP}$ and $2 \mathrm{HP}$ problems on interval graphs remains an open question.

Motivated by the above issues, we state a variant of the path cover problem, namely, the 1-fixed-endpoint path cover (1PC) problem, which generalizes the $1 \mathrm{HP}$ problem.

Problem 1PC Given a graph $G$ and a vertex $u \in V(G)$, a 1-fixed-endpoint path cover of the graph $G$ with respect to $u$ is a path cover of $G$ such that the vertex $u$ is an endpoint of a path in the path cover; a minimum 1-fixed-endpoint path cover of $G$ with respect to $u$ is a 1 -fixed-endpoint path cover of $G$ with minimum cardinality; the 1-fixed-endpoint path cover problem (1PC) is to find a minimum 1-fixed-endpoint path cover of the graph $G$.

Contribution In this paper, we study the complexity status of the 1-fixed-endpoint path cover problem (1PC) on the class of interval graphs [7, 12], and show that this problem can be solved in polynomial time. The proposed algorithm runs in $O\left(n^{3}\right)$ time on an interval graph $G$ on $n$ vertices and $m$ edges and requires linear space. The proposed algorithm for the 1PC problem can also be used to solve the 1HP problem on interval graphs within the same time and space complexity. Using our algorithm for the 1PC problem and a simple reduction described by Müller in [20], we solve the HP problem on a $X$-convex graph $G(X, Y, E)$ with $|Y|-|X|=1$, which was left open in [31]. We also show that the 1 HP problem on a biconvex graph $G$ is solvable in $O\left(n^{3}\right)$ time. Figure 1 shows a diagram of class inclusions for a number of graph classes, subclasses of comparability and chordal graphs, and the current complexity status of the $1 \mathrm{HP}$ problem on these classes; for definitions of the classes shown, see $[7,12]$.

Related Work Interval graphs form an important class of perfect graphs [12] and many problems that are NP-complete on arbitrary graphs are shown to admit polynomial time algorithms on this class $[2,12,18]$. Both Hamiltonian Circuit (HC) and Hamiltonian Path (HP) problems are polynomially solvable for the class of interval and proper interval graphs. Keil introduced a linear-time algorithm for the HC problem on interval graphs [18] and Arikati and Rangan [2] presented a linear-time algorithm for the minimum path cover problem on interval graphs. Bertossi [5] proved that a proper interval graph has a Hamiltonian path if and only if it is connected. He also gave an $O(n \log n)$ algorithm for finding a Hamiltonian circuit in a proper interval graph. Recently, Asdre and Nikolopoulos proposed a linear-time algorithm for the $k$-fixed-endpoint path cover problem $(k \mathrm{PC})$ on cographs and on proper interval 


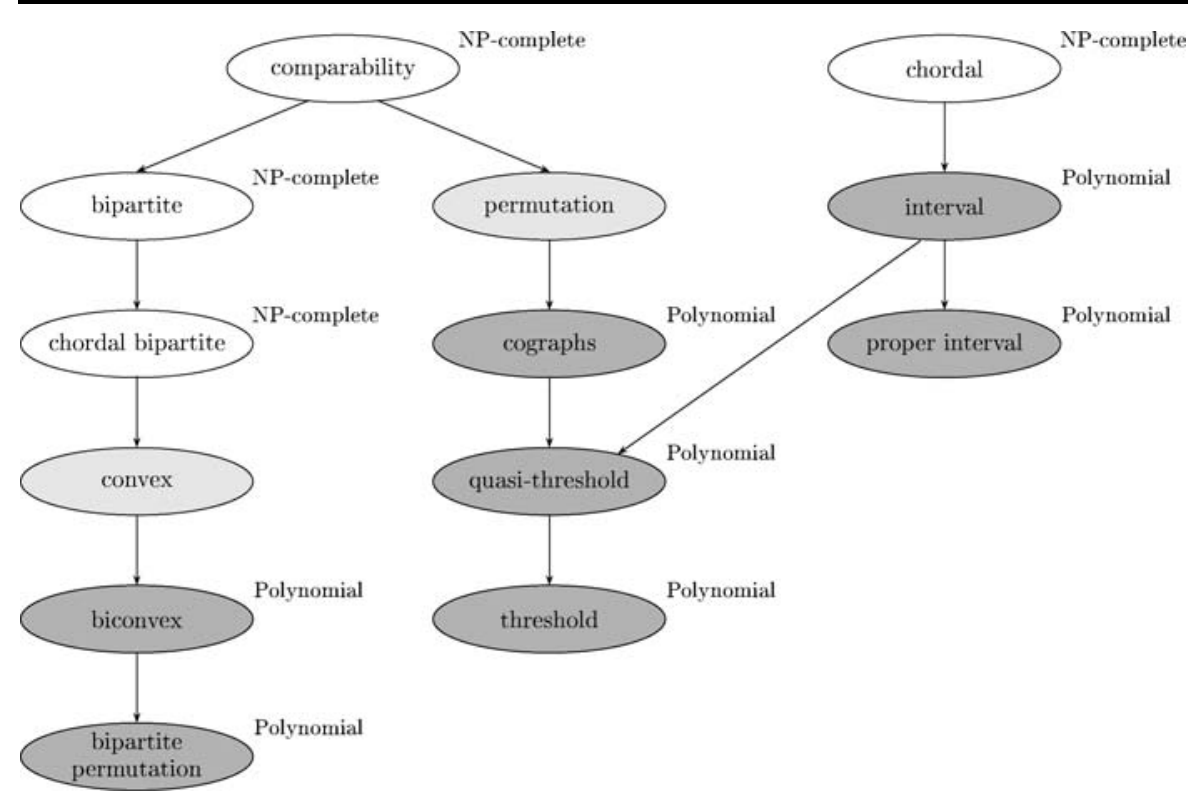

Fig. 1 The complexity status (NP-complete, unknown, polynomial) of the 1HP problem for some graph subclasses of comparability and chordal graphs. $A \rightarrow B$ indicates that class $A$ contains class $B$

graphs [3, 4]. Furthermore, Lin et al. [19] proposed an optimal algorithm for the path cover problem on cographs while Nakano et al. [21] proposed an optimal parallel algorithm which finds and reports all the paths in a minimum path cover of a cograph in $O(\log n)$ time using $O(n / \log n)$ processors on a PRAM model. Hsieh et al. [14] presented an $O(n+m)$-time sequential algorithm for the Hamiltonian problem on a distance-hereditary graph and also proposed a parallel implementation of their algorithm which solves the problem in $O(\log n)$ time using $O((n+m) / \log n)$ processors on a PRAM model. A unified approach to solving the Hamiltonian problems on distance-hereditary graphs was presented in [15], while Hsieh [13] presented an efficient parallel strategy for the 2HP problem on the same class of graphs. Algorithms for the path cover problem on other classes of graphs were proposed in $[16,22,28]$.

Road Map The paper is organized as follows. In Sect. 2 we establish the notation and related terminology, and we present background results. In Sect. 3 we describe our algorithm for the 1PC problem, while in Sect. 4 we prove its correctness and compute its time and space complexity. Section 5 presents some related results and in Sect. 6 we conclude the paper and discuss possible future extensions.

\section{Theoretical Framework}

We consider finite undirected graphs with no loops or multiple edges. For a graph $G$, we denote its vertex and edge set by $V(G)$ and $E(G)$, respectively. Let $S$ be a subset of the vertex set of a graph $G$. Then, the subgraph of $G$ induced by $S$ is denoted by $G[S]$. Furthermore, we say that a vertex $v$ sees vertex $v^{\prime}$ if and only if $v v^{\prime} \in E(G)$. 
A graph $G$ is an interval graph if its vertices can be put in a one-to-one correspondence with a family $F$ of intervals on the real line such that two vertices are adjacent in $G$ if and only if their corresponding intervals intersect. $F$ is called an intersection model for $G$ [2]. Interval graphs find applications in genetics, molecular biology, archeology, and storage information retrieval [12]. Interval graphs form an important class of perfect graphs [12] and many problems that are NP-complete on arbitrary graphs are shown to admit polynomial time algorithms on this class $[2,12,18]$. The class of interval graphs is hereditary, that is, every induced subgraph of an interval graph $G$ is also an interval graph. We state the following numbering for the vertices of an interval graph proposed in [24].

Lemma 2.1 (Ramalingam and Rangan [24]) The vertices of any interval graph $G$ can be numbered with integers $1,2, \ldots,|V(G)|$ such that if $i<j<k$ and $i k \in E(G)$ then $j k \in E(G)$.

As shown in [24], the numbering of Lemma 2.1, which results from numbering the intervals after sorting them on their right ends [2], can be obtained in linear time, that is, $O(m+n)$ time. An ordering of the vertices according to this numbering is found to be quite useful in solving many problems on interval graphs $[2,24]$. Throughout the paper, the vertex numbered with $i$ will be denoted by $v_{i}, 1 \leq i \leq n$, and such an ordering will be denoted by $\pi$. We say that $v_{i}<v_{j}$ if $i<j, 1 \leq i, j \leq n$.

Let $G$ be an interval graph with vertex set $V(G)$ and edge set $E(G), \mathcal{T}$ be a set containing a single vertex of $V(G)$, and let $\mathcal{P}_{\mathcal{T}}(G)$ be a minimum 1-fixed-endpoint path cover of $G$ with respect to $\mathcal{T}$ of size $\lambda_{\mathcal{T}}(G)$ (or $\lambda_{\mathcal{T}}$ for short); recall that the size of $\mathcal{P}_{\mathcal{T}}(G)$ is the number of paths it contains. The vertex belonging to the set $\mathcal{T}$ is called terminal vertex, and the set $\mathcal{T}$ is called the terminal set of $G$, while those of $V(G)-\mathcal{T}$ are called non-terminal vertices. Thus, the set $\mathcal{P}_{\mathcal{T}}(G)$ contains two types of paths, which we call terminal and non-terminal paths: a terminal path $P_{t}$ is a path having the terminal vertex as an endpoint and a non-terminal path $P_{f}$ is a path having both its endpoints in $V(G)-\mathcal{T}$. The set of the non-terminal paths in a minimum 1PC of the graph $G$ is denoted by $N$, while $T$ denotes the set containing the terminal path. Clearly, $|T|=1$ and $\lambda_{\mathcal{T}}=|N|+1$.

Our algorithm for computing a 1PC of an interval graph is based on a greedy principle, visiting the vertices according to the ordering $\pi=\left(v_{1}, v_{2}, \ldots, v_{k}, \ldots, v_{n}\right)$, and uses three operations on the paths of a 1PC of $G[S]$, where $S=\left\{v_{1}, v_{2}, \ldots, v_{k}\right\}$, $1 \leq k<n$. These three operations, namely connect, insert and bridge operations, are described below and are illustrated in Fig. 2:

- Connect operation. Let $v_{i}$ be a non-terminal endpoint of a path $P$ of $\mathcal{P}_{\mathcal{T}}(G[S])$ and let $v_{k+1}$ be a non-terminal or a terminal vertex such that $v_{k+1}$ sees $v_{i}$. We say that we connect vertex $v_{k+1}$ to the path $P$, or, equivalently, to the vertex $v_{i}$, if we extend the path $P$ by adding an edge which joins vertex $v_{k+1}$ with vertex $v_{i}$.

- Insert operation. Let $P=\left(\ldots, v_{i}, v_{j}, \ldots\right), i \neq j, i, j \in[1, k]$, be a path of $\mathcal{P}_{\mathcal{T}}(G[S])$ and let $v_{k+1}$ be a non-terminal vertex such that $v_{k+1}$ sees $v_{i}$ and $v_{j}$. We say that we insert vertex $v_{k+1}$ into $P$ (between $v_{i}$ and $v_{j}$ ), if we replace the path $P$ with the path $P^{\prime}=\left(\ldots, v_{i}, v_{k+1}, v_{j}, \ldots\right)$. 
Fig. 2 Illustrating

(a) connect, (b) insert, and

(c) bridge operations;

$P, P_{1}, P_{2} \in \mathcal{P}_{\mathcal{T}}(G[S])$
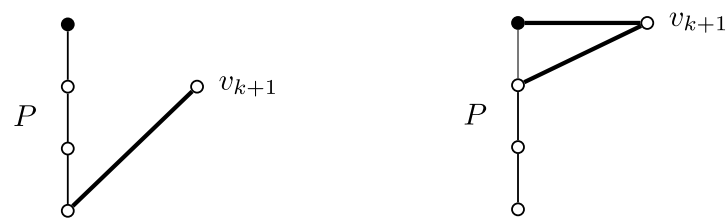

(a)

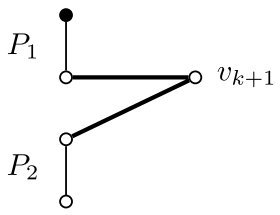

(c)

- Bridge operation. Let $P_{1}$ and $P_{2}$ be two paths of $\mathcal{P}_{\mathcal{T}}(G[S])$ and let $v_{k+1}$ be a non-terminal vertex that sees at least one non-terminal endpoint of $P_{1}$ and at least one of $P_{2}$. We say that we bridge the two paths $P_{1}$ and $P_{2}$ using vertex $v_{k+1}$ if we connect $v_{k+1}$ with a non-terminal endpoint of $P_{1}$ and a non-terminal endpoint of $P_{2}$.

Let $P$ be a path of $\mathcal{P}_{\mathcal{T}}(G)$ and let $v_{i}$ and $v_{j}$ be its endpoints. We say that $v_{i}$ is the left (resp. right) endpoint of the path and $v_{j}$ is the right (resp. left) endpoint of the path if $v_{i}<v_{j}$ (resp. $v_{j}<v_{i}$ ). Throughout the paper, a trivial path (i.e. a path consisting of one vertex) is considered to have two endpoints, while a trivial path consisting of the terminal vertex $u \in \mathcal{T}$ is considered to have one terminal endpoint and one non-terminal endpoint.

Let $G$ be an interval graph on $n$ vertices and let $\mathcal{P}_{\mathcal{T}}(G)$ be a minimum 1 PC of size $\lambda_{\mathcal{T}}$. Since a trivial path is considered to have two endpoints, the number of endpoints in $\mathcal{P}_{\mathcal{T}}(G)$ is $2 \lambda_{\mathcal{T}}$. For each vertex $v_{i}$ we denote by $d\left(v_{i}\right)$ the number of neighbors of $v_{i}$ in $\mathcal{P}_{\mathcal{T}}(G)$; that is, $d\left(v_{i}\right) \in\{0,1,2\}$. We call $d$-connectivity of $\mathcal{P}_{\mathcal{T}}(G)$ the sum of $d\left(v_{1}\right), d\left(v_{2}\right), \ldots, d\left(v_{n}\right)$. It is easy to see that $\sum_{i=1}^{n} d\left(v_{i}\right)=2\left(n-\lambda_{\mathcal{T}}\right)$. Clearly, any minimum $1 \mathrm{PC} \mathcal{P}_{\mathcal{T}}(G)$ has d-connectivity equal to $2\left(n-\lambda_{\mathcal{T}}\right)$.

\section{The Algorithm}

In this section we present an algorithm for the 1PC problem on interval graphs. Our algorithm takes as input an interval graph $G$ on $n$ vertices and $m$ edges and a set $\mathcal{T}=\{u\}$ containing the terminal vertex $u \in V(G)$, and computes a minimum 1PC $\mathcal{P}_{\mathcal{T}}(G)$ of $G$ in $O\left(n^{3}\right)$ time. The algorithm is based on a greedy principle to extend a path of a minimum 1PC using operations on the left and right endpoints of its paths and properties of the graph $G\left[\left\{v_{1}, v_{2}, \ldots, v_{i}\right\}-\{u\}\right], 1 \leq i \leq n$; if a vertex sees the two endpoints of only one non-terminal path $P$, it is connected to the left endpoint of the path $P$. Furthermore, for each vertex $v_{i}, 1 \leq i<j$, we denote by $\varepsilon_{i}^{(j)}$ the number of endpoints $v_{\kappa}$ belonging to different paths in $\mathcal{P}_{\mathcal{T}}\left(G\left[v_{1}, v_{2}, \ldots, v_{j}\right]\right)$ with index $\kappa \in(i, j]$. We also define $\varepsilon_{i}^{(i)}=0$ and $\varepsilon_{0}^{(i)}=\lambda_{\mathcal{T}}\left(G\left[v_{1}, v_{2}, \ldots, v_{i}\right]\right), 1 \leq i \leq n$. 


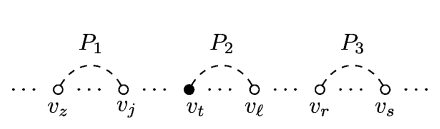

(a)

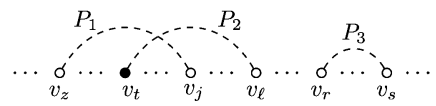

(c)

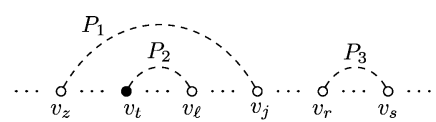

(b)

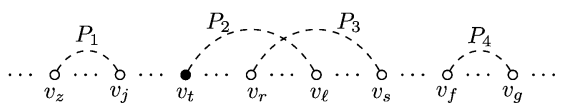

(d)

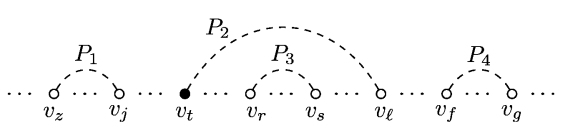

(e)

Fig. 3 Illustrating some cases of the bridge operation

Before describing our algorithm, let us present the operation bridge in detail. In most cases we bridge two paths that have the leftmost non-terminal endpoints. Suppose that when vertex $v_{i}$ is processed it sees at least one non-terminal endpoint of a non-terminal path $P_{1}$, say, $v_{j}$, and at least the non-terminal endpoint of the terminal path $P_{2}$, say, $v_{\ell}$, and both endpoints of a non-terminal path $P_{3}$, say, $v_{r}$ and $v_{s}$. Let $v_{z}, v_{t}$ and $v_{r}$ be the left endpoints and $v_{j}, v_{\ell}$ and $v_{s}$ be the right endpoints of the paths $P_{1}, P_{2}$ and $P_{3}$, respectively. There exist three cases where, in order to obtain the maximum possible value for every $\varepsilon_{i}^{(j)}$, we do not bridge two paths through the leftmost non-terminal endpoints (see Fig. 3(a)-(c)). In these three cases the bridge operation works as follows:

(a) $v_{z}<v_{j}<v_{t}<v_{\ell}<v_{r}<v_{s}$ : we bridge $P_{1}$ and $P_{3}$ through $v_{z}$ (or, $v_{j}$ if $v_{z} \notin$ $\left.N\left(v_{i}\right)\right)$ and $v_{r}$.

(b) $v_{z}<v_{t}<v_{\ell}<v_{j}<v_{r}<v_{s}$ : if $v_{z} \notin N\left(v_{i}\right)$ we bridge $P_{2}$ and $P_{3}$ through $v_{\ell}$ and $v_{r}$; otherwise, we bridge $P_{1}$ and $P_{2}$ through $v_{z}$ and $v_{\ell}$.

(c) $v_{z}<v_{t}<v_{j}<v_{\ell}<v_{r}<v_{s}$ : we bridge $P_{1}$ and $P_{3}$ through $v_{z}$ (or, $v_{j}$ if $v_{z} \notin$ $\left.N\left(v_{i}\right)\right)$ and $v_{r}$.

Suppose now that $P_{1}$ is a non-terminal path having $v_{z}$ and $v_{j}$ as its left and right endpoints, respectively, $P_{2}$ is the terminal path with left endpoint $v_{t}$ and right endpoint $v_{\ell}$. Also, let $P_{3}$ be a non-terminal path with left and right endpoints $v_{r}$ and $v_{s}$, respectively, and $P_{4}$ a non-terminal path with left and right endpoints $v_{f}$ and $v_{g}$, respectively (see Fig. 3(d)-(e)). We distinguish the following two cases:

(d) $v_{z}<v_{j}<v_{t}<v_{r}<v_{\ell}<v_{s}<v_{f}<v_{g}$ : if $v_{z} \in N\left(v_{i}\right)$ or $v_{j} \in N\left(v_{i}\right)$ we bridge $P_{1}$ and $P_{3}$ through $v_{z}\left(v_{j}\right.$ if $\left.v_{z} \notin N\left(v_{i}\right)\right)$ and $v_{r}$. If $v_{\ell} \in N\left(v_{i}\right)$ and $v_{r} \notin N\left(v_{i}\right)$ we bridge $P_{2}$ and $P_{4}$ through $v_{\ell}$ and $v_{f}$.

(e) $v_{z}<v_{j}<v_{t}<v_{r}<v_{s}<v_{\ell}<v_{f}<v_{g}$ : if $v_{z} \in N\left(v_{i}\right)$ or $v_{j} \in N\left(v_{i}\right)$ we bridge $P_{1}$ and $P_{3}$ through $v_{i}\left(v_{j}\right.$ if $\left.v_{z} \notin N\left(v_{i}\right)\right)$ and $v_{r}$; otherwise, we bridge $P_{3}$ and $P_{4}$ through $v_{r}\left(v_{s}\right.$ if $\left.v_{r} \notin N\left(v_{i}\right)\right)$ and $v_{f}$.

Figure 3 presents cases (a)-(e). Suppose that we have the two paths $P_{2}$ and $P_{3}$ of case (e) and vertex $v_{i}$ sees both $v_{r}$ and $v_{s}$, that is, $P_{2}=\left(v_{t}, \ldots, v_{a}, v_{b}, v_{c}, \ldots, v_{\ell}\right)$ 
and $P_{3}=\left(v_{r}, \ldots, v_{s}\right)$, where $v_{a}<v_{s}<v_{b}$ and $v_{s}<v_{c}$. Then, the bridge operation constructs the path $P=\left(v_{t}, \ldots, v_{a}, v_{b}, v_{s}, \ldots, v_{r}, v_{i}, v_{c}, \ldots, v_{\ell}\right)$. Suppose now that we have the two paths $P_{1}$ and $P_{2}$ of case (c) and vertex $v_{i}$ sees all vertices with index greater or equal to $z$, that is, $P_{1}=\left(v_{z}, \ldots, v_{j}\right)$ and $P_{2}=$ $\left(v_{t}, \ldots, v_{a}, v_{b}, v_{c}, \ldots, v_{\ell}\right)$, where $v_{a}<v_{j}<v_{b}$ and $v_{j}<v_{c}$. Then, the bridge operation constructs the path $P=\left(v_{t}, \ldots, v_{a}, v_{b}, v_{j}, \ldots, v_{z}, v_{i}, v_{c}, \ldots, v_{\ell}\right)$. Suppose that there exist two paths $P_{2}$ and $P_{3}$ as in case (d) and vertex $v_{i}$ sees all vertices with index $k, d \leq k$, where $r<d \leq \ell$, that is, $P_{2}=\left(v_{t}, \ldots, v_{a}, v_{b}, v_{c}, \ldots, v_{\ell}\right)$ and $P_{3}=\left(v_{r}, \ldots, v_{s}\right)$, where $v_{a}<v_{r}<v_{b}$ and $v_{r}<v_{c}$. If $d<c$ then the bridge operation constructs the path $P=\left(v_{t}, \ldots, v_{a}, v_{b}, v_{r}, \ldots, v_{s}, v_{i}, v_{c}, \ldots, v_{\ell}\right)$; otherwise, it constructs the path $P=\left(v_{t}, \ldots, v_{a}, v_{b}, v_{r}, \ldots, v_{s}, v_{i}, v_{\ell}, \ldots, v_{c}\right)$. If there exist two paths $P_{1}$ and $P_{2}$ as in case (b) and vertex $v_{i}$ sees all vertices with index greater or equal to $z$, that is, $P_{1}=\left(v_{z}, \ldots, v_{a}, v_{b}, v_{c}, \ldots, v_{j}\right)$ and $P_{2}=$ $\left(v_{t}, \ldots, v_{\ell}\right)$, where $v_{a}<v_{\ell}<v_{b}$ and $v_{\ell}<v_{c}$, then the bridge operation constructs the path $P=\left(v_{t}, \ldots, v_{\ell}, v_{b}, v_{a}, \ldots, v_{z}, v_{i}, v_{c}, \ldots, v_{j}\right)$, if $c<j$, or the path $P=$ $\left(v_{t}, \ldots, v_{\ell}, v_{b}, v_{a}, \ldots, v_{z}, v_{i}, v_{j}, \ldots, v_{c}\right)$, if $j<c$.

We next describe the operation new_path of our algorithm which creates a new path when the vertex $v_{i}$ is processed. There exist three cases where operation new_path creates a new non-trivial path while in all other cases it creates a new trivial path. Suppose that $v_{i}$ sees an internal vertex $v_{j}$ belonging to a path $P=\left(v_{s}, \ldots, v_{r}, v_{j}, v_{\ell}, \ldots, v_{t}\right)$ such that $v_{r}<v_{s}<v_{t}<v_{\ell}<v_{j}$. We remove the edge $v_{j} v_{\ell}$ from $P$ and we obtain $P_{1}=\left(v_{s}, \ldots, v_{r}, v_{j}\right)$ and $P_{2}=\left(v_{t}, \ldots, v_{\ell}\right)$. Then, we connect $v_{i}$ to $v_{j}$. The case where $v_{j} v_{s} \in E(G)$ and $v_{j} v_{r} \notin E(G)$ is similar. If $v_{i}$ sees an internal vertex $v_{j}$ belonging to a path $P=\left(v_{r}, \ldots, v_{s}, v_{j}, v_{\ell}, \ldots, v_{t}\right)$ such that $v_{r}<v_{t}<v_{\ell}<v_{s}<v_{j}$, we remove the edge $v_{s} v_{j}$ from $P$ and we obtain $P_{1}=\left(v_{r}, \ldots, v_{s}\right)$ and $P_{2}=\left(v_{t}, \ldots, v_{\ell}, v_{j}\right)$. Then, we connect $v_{i}$ to $v_{j}$. Suppose now that $v_{i}$ sees an internal vertex $v_{j}$ belonging to a path $P=\left(v_{\ell}, \ldots, v_{s}, v_{j}, v_{r}, \ldots, v_{t}\right)$ such that $v_{\ell}<v_{t}<v_{s}<v_{r}<v_{j}$. We remove the edge $v_{j} v_{r}$ from $P$ and we obtain $P_{1}=\left(v_{\ell}, \ldots, v_{s}, v_{j}\right)$ and $P_{2}=\left(v_{t}, \ldots, v_{r}\right)$. Then, we connect $v_{i}$ to $v_{j}$. The above cases, where the operation new_path creates a new non-trivial path, are described below:

(a) $v_{r}<v_{s}<v_{t}<v_{\ell}<v_{j}$. We create paths $P_{1}=\left(v_{s}, \ldots, v_{r}, v_{j}, v_{i}\right)$ and $P_{2}=$ $\left(v_{t}, \ldots, v_{\ell}\right)$. The case where $v_{j} v_{s} \in E(G)$ and $v_{j} v_{r} \notin E(G)$ is similar.

(b) $v_{r}<v_{t}<v_{\ell}<v_{s}<v_{j}$. We create paths $P_{1}=\left(v_{r}, \ldots, v_{s}\right)$ and $P_{2}=\left(v_{t}, \ldots\right.$, $\left.v_{\ell}, v_{j}, v_{i}\right)$.

(c) $v_{\ell}<v_{t}<v_{s}<v_{r}<v_{j}$. We create paths $P_{1}=\left(v_{\ell}, \ldots, v_{s}, v_{j}, v_{i}\right)$ and $P_{2}=$ $\left(v_{t}, \ldots, v_{r}\right)$.

Note that, the rightmost endpoint of a path in $\mathcal{P}_{\mathcal{T}}\left(G\left[\left\{v_{1}, v_{2}, \ldots, v_{i-1}\right\}\right]\right)$ is $v_{t}$ and, thus, $\varepsilon_{t}^{(i-1)}=0$. The $1 \mathrm{PC} \mathcal{P}_{\mathcal{T}}(G)$ of the graph $G$ in each of the above cases contains two new endpoints, vertices $v_{i}$ and $v_{k^{\prime}}$ such that $t<k^{\prime}$; thus, $\varepsilon_{t}^{(i)}=2$. Figure 4 presents the above cases.

The operation connect_break of our algorithm is similar to the operation new_path. Specifically, suppose that in the above cases (a)-(c) there exists a path $P=\left(v_{a}, \ldots, v_{b}\right)$ such that $v_{j}<v_{a}<v_{b}<v_{i}$. Then, the operation connect_break works similarly to the operation new_path; the only difference is that $v_{i}$ is also connected to $v_{a}$. 
(a)
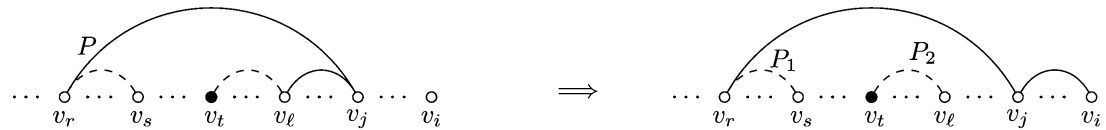

(b)
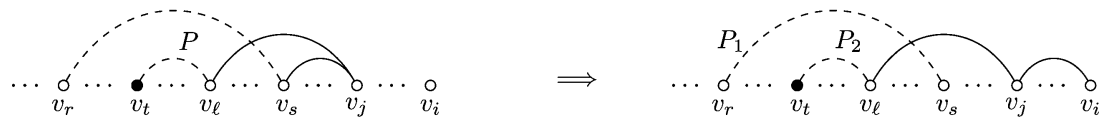

(c)
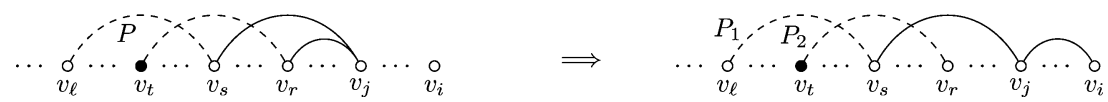

Fig. 4 Illustrating some cases of the new_path operation

We next present our algorithm which works as follows.

Algorithm 1PC_INTERVAL

Input: an interval graph $G$ on $n$ vertices and $m$ edges and a vertex $u \in V(G)$;

Output: a minimum $1 \mathrm{PC} \mathcal{P}_{\mathcal{T}}(G)$ of the interval graph $G$, where $\mathcal{T}=\{u\}$;

1. Construct the ordering $\pi$ of the vertices of $G$. Let $t$ be the index of the terminal vertex $u$;

2. $\lambda_{\mathcal{T}} \leftarrow 1 ; P_{\lambda_{\mathcal{T}}} \leftarrow\left(v_{1}\right) ; \varepsilon_{0}^{(1)} \leftarrow 1$;

$v_{1}$ is the left and right endpoint of the path $P_{\lambda_{\mathcal{T}}}$;

3. for $i=2$ to $n$ do

if $i \neq t$ then execute the procedure process_non_terminal;

else execute the procedure process_terminal;

4. $\mathcal{P}_{\mathcal{T}}(G)=\left\{P_{1}, P_{2}, \ldots, P_{\lambda_{\mathcal{T}}}\right\}$;

End_of_Algorithm 1PC_INTERVAL.

The procedures process_non_terminal and process_terminal are described in Figs. 5 and 6, respectively. We point out that, if no vertex is specified as the terminal vertex then Algorithm 1PC_INTERVAL returns a minimum path cover of $G$.

We next show that, if $\lambda_{\mathcal{T}}(G)$ is the size of a minimum 1PC of $G$ with respect to $\mathcal{T}=\left\{v_{t}\right\}$ then the size of a minimum $1 \mathrm{PC}$ of $G-\left\{v_{t}\right\}$ is either $\lambda_{\mathcal{T}}(G)$ or $\lambda_{\mathcal{T}}(G)-1$.

Lemma 3.1 Let $G$ be an interval graph and $\lambda_{\mathcal{T}}(G)$ be the size of a minimum 1PC of $G$ with respect to $\mathcal{T}=\left\{v_{t}\right\}$. The size of a minimum PC of $G-\left\{v_{t}\right\}$ is either $\lambda_{\mathcal{T}}(G)$ or $\lambda_{\mathcal{T}}(G)-1$.

Proof Suppose that the size of a minimum PC of $G-\left\{v_{t}\right\}$ is at least $\lambda \mathcal{T}(G)+1$. Since a terminal vertex cannot decrease the size of a minimum 1PC, we have $\lambda_{\mathcal{T}}(G) \geq$ $\lambda_{\mathcal{T}}\left(G-\left\{v_{t}\right\}\right)$. Thus, $\lambda_{\mathcal{T}}(G) \geq \lambda_{\mathcal{T}}(G)+1$, a contradiction. Suppose now that the size of a minimum $\mathrm{PC} \mathcal{P}_{\mathcal{T}}\left(G-\left\{v_{t}\right\}\right)$ of $G-\left\{v_{t}\right\}$ is at most $\lambda_{\mathcal{T}}(G)-2$. Then, adding a trivial path containing vertex $v_{t}$ to $\mathcal{P}_{\mathcal{T}}\left(G-\left\{v_{t}\right\}\right)$ results to a $1 \mathrm{PC}$ of $G$ of size $\lambda_{\mathcal{T}}(G)-1$, a contradiction. 
Process_non_terminal

\{the vertex $v_{i}$ of $\pi=\left(v_{1}, v_{2}, \ldots, v_{i}, \ldots, v_{n}\right)$ is not terminal, that is, $\left.i \neq t\right\}$

$\left\{\right.$ let $N^{\prime}\left(v_{i}\right)=\left\{v_{j} \in N\left(v_{i}\right): j<i\right\}, v_{j}$ is the leftmost neighbor of $\left.v_{i} \in N^{\prime}\left(v_{i}\right)\right\}$

- if $N^{\prime}\left(v_{i}\right) \neq \emptyset$ and $\varepsilon_{j-1}^{(i-1)} \geq 2$ then

if at least two endpoints are non-terminal vertices then bridge; $\lambda_{\mathcal{T}} \leftarrow \lambda_{\mathcal{T}}-1$;

else $\left\{\varepsilon_{j-1}^{(i-1)}=2\right.$ and one endpoint is the terminal vertex $\left.v_{t}.\right\}$

if 1PC_INTERVAL on $G\left[\left\{v_{1}, \ldots, v_{i-1}\right\}-\left\{v_{t}\right\}\right]$ returns a path cover of $\lambda_{\mathcal{T}}-1$ paths then $\left\{\right.$ let $\mathcal{P}_{\mathcal{T}}\left(G\left[\left\{v_{1}, \ldots, v_{i-1}\right\}-\left\{v_{t}\right\}\right]\right)$ be such a path cover $\}$

connect $v_{i}$ to the leftmost endpoint of $\mathcal{P}_{\mathcal{T}}\left(G\left[\left\{v_{1}, \ldots, v_{i-1}\right\}-\left\{v_{t}\right\}\right]\right)$;

connect $v_{t}$ to $v_{i}$

$\lambda_{\mathcal{T}} \leftarrow \lambda_{\mathcal{T}}-1$

else-if 1PC_InTERVAL on $G\left[\left\{v_{1}, \ldots, v_{i-1}\right\}-\left\{v_{t}\right\}\right]$ returns a path cover of $\lambda_{\mathcal{T}}$ paths then

if during the execution of 1PC_INTERVAL on $G\left[\left\{v_{1}, \ldots, v_{i-1}\right\}\right]$ and $v_{t}$ the following hold:

(i) there exists $v_{b}$ such that $v_{t} \in N^{\prime}\left(v_{b}\right)$;

(ii) breaking $v_{t}$ from its path and connecting $v_{t}$ to $v_{b}$ results to a $1 \mathrm{PC}$ $\mathcal{P}_{\mathcal{T}}^{\prime}\left(G\left[\left\{v_{1}, \ldots, v_{i-1}\right\}\right]\right)$ of $\lambda_{\mathcal{T}}$ paths;

(iii) $\varepsilon_{j-1}^{(i-1)}=2$ and both endpoints are non-terminal vertices

then $\mathcal{P}_{\mathcal{T}}\left(G\left[\left\{v_{1}, \ldots, v_{i-1}\right\}\right]\right) \leftarrow \mathcal{P}_{\mathcal{T}}^{\prime}\left(G\left[\left\{v_{1}, \ldots, v_{i-1}\right\}\right]\right)$; bridge; $\lambda_{\mathcal{T}} \leftarrow \lambda_{\mathcal{T}}-1$; else connect_break;

- if $N^{\prime}\left(v_{i}\right) \neq \emptyset$ and $\varepsilon_{j-1}^{(i-1)}=1$ and the endpoint $v_{f}, j \leq f \leq i-1$, is non terminal then if $v_{i}$ sees an internal vertex $v_{s}$ then connect_break;

else connect $v_{i}$ to the leftmost non-terminal endpoint;

- if $N^{\prime}\left(v_{i}\right)=\emptyset$ or $\varepsilon_{j-1}^{(i-1)}=0$ or

$\left(\varepsilon_{j-1}^{(i-1)}=1\right.$ and the endpoint $v_{t}, j \leq t \leq i-1$, is the terminal vertex $)$ then

if $v_{i}$ has two consecutive neighbors into a path then insert $v_{i}$ into the path;

else-if $v_{i}$ sees an internal vertex $v_{s}$ then

if $v_{s} v_{a}$ is an edge of a path $P_{k}$ and $v_{a}$ sees an endpoint $v_{b}$ of a path $P_{k^{\prime}}, k \neq k^{\prime}$

then remove the edge $v_{s} v_{a}$ of $P$; connect $v_{a}$ to $v_{b}$; connect $v_{i}$ to $v_{j}$;

else new_path; $\lambda_{\mathcal{T}} \leftarrow \lambda_{\mathcal{T}}+1$;

else $\lambda_{\mathcal{T}} \leftarrow \lambda_{\mathcal{T}}+1 ; P_{\lambda_{\mathcal{T}}} \leftarrow\left(v_{i}\right)$;

- update the values $\varepsilon_{k}^{(i)}, 0 \leq k \leq i$, and the left and right endpoints of the paths;

Fig. 5 The procedure process_non_terminal of the Algorithm 1PC_INTERVAL

Process_terminal

\{the vertex $v_{i}$ of $\pi=\left(v_{1}, v_{2}, \ldots, v_{i}, \ldots, v_{n}\right)$ is terminal, that is, $\left.i=t\right\}$

- if $\varepsilon_{j-1}^{(i-1)} \geq 1$ then connect $v_{i}$ to the leftmost endpoint of $\mathcal{P}_{\mathcal{T}}\left(G\left[\left\{v_{1}, \ldots, v_{i-1}\right\}\right]\right)$; else $\lambda_{\mathcal{T}} \leftarrow \lambda_{\mathcal{T}}+1 ; P_{\lambda_{\mathcal{T}}} \leftarrow\left(v_{i}\right)$;

- update the values $\varepsilon_{k}^{(i)}, 0 \leq k \leq i$, and the left and right endpoints of the paths;

Fig. 6 The procedure process_terminal of the Algorithm 1PC_INTERVAL 
Concerning the ordering of the endpoints of the paths of the 1PC constructed by Algorithm 1PC_INTERVAL, we prove the following lemma.

Lemma 3.2 Let $G$ be an interval graph with no terminal vertex. Let $P_{S}, 1 \leq$ $s \leq \lambda_{\mathcal{T}}$, be a path in the $1 \mathrm{PC} \mathcal{P}_{\mathcal{T}}(G)$ of the graph $G$ constructed by Algorithm 1PC_INTERVAL and let $v_{i}$ and $v_{j}$ be the left and right endpoints of $P_{s}$, respectively. Then, there is no path $P_{t} \in \mathcal{P}_{\mathcal{T}}(G), 1 \leq t \leq \lambda_{\mathcal{T}}, t \neq s$, such that $v_{i}<v_{k}<v_{j}$ or $v_{i}<v_{\ell}<v_{j}$, where $v_{k}$ and $v_{\ell}$ are the left and right endpoints of $P_{t}$, respectively.

Proof Let $P_{s}, 1 \leq s \leq \lambda_{\mathcal{T}}$ be a path in the $1 \mathrm{PC} \mathcal{P}_{\mathcal{T}}(G)$ constructed by Algorithm 1PC_INTERVAL and let $v_{i}$ and $v_{j}$ be its left and right endpoints, respectively. Let $P_{t} \in \mathcal{P}_{\mathcal{T}}(G), 1 \leq t \leq \lambda_{\mathcal{T}}, t \neq s$, and let $v_{k}$ and $v_{\ell}$ be its left and right endpoints, respectively. Suppose that $v_{i}<v_{k}<v_{j}$. Since $v_{i}$ and $v_{j}$ are the endpoints of $P_{s}$ and $v_{i}<v_{k}<v_{j}$, the path $P_{s}$ contains at least one edge, say, $v_{a} v_{b}$, such that $v_{a}<v_{k}<v_{b}$. Clearly, vertices $v_{a}$ and $v_{b}$ are non-terminal vertices. Since $v_{a} v_{b} \in E(G)$, we also have $v_{k} v_{b} \in E(G)$. Then, according to Algorithm 1PC_INTERVAL, when vertex $v_{b}$ is processed, vertex $v_{k}$ is an endpoint of the path $P_{t}$, and, thus, $v_{b}$ bridges the paths $P_{s}$ and $P_{t}$ through vertices $v_{a}$ and $v_{k}$, a contradiction. Similarly, we can prove that $v_{\ell}<v_{i}$ or $v_{\ell}>v_{j}$.

Using similar arguments we can prove the following lemma.

Lemma 3.3 Let $G$ be an interval graph containing a terminal vertex. Let $P_{S}$, $1 \leq s \leq \lambda_{\mathcal{T}}$, be a non-terminal path in the $1 \mathrm{PC} \mathcal{P}_{\mathcal{T}}(G)$ of the graph $G$ constructed by Algorithm 1PC_INTERVAL and let $v_{i}$ and $v_{j}$ be the left and right endpoints of $P_{s}$, respectively. Then, there is no non-terminal path $P_{t} \in \mathcal{P}_{\mathcal{T}}(G), 1 \leq t \leq \lambda_{\mathcal{T}}, t \neq s$, such that $v_{i}<v_{k}<v_{j}$ or $v_{i}<v_{\ell}<v_{j}$, where $v_{k}$ and $v_{\ell}$ are the left and right endpoints of $P_{t}$, respectively.

\section{Correctness and Time Complexity}

Let $G$ be an interval graph on $n$ vertices and $m$ edges and let $\mathcal{T}$ be a subset of $V(G)$ containing a single vertex $v \in V(G)$. In order to prove the correctness of Algorithm 1PC_INTERVAL, we use induction on $n$. We also prove a property of the minimum $1 \mathrm{PC} \mathcal{P}_{\mathcal{T}}(G)$ of $G$ constructed by our algorithm, namely, Property 1 PC-on- $H$ :

Property 1PC-on-H Let $H$ be an interval graph with vertex set $V(H)=\left\{u_{1}, u_{2}\right.$, $\left.\ldots, u_{n}\right\}$, let $\mathcal{P}_{\mathcal{T}}(H)$ be a minimum $1 \mathrm{PC}$ of the graph $H$, and let $\varepsilon_{i}^{\left({ }^{(n)}\right.}$ be the number of endpoints $v_{\kappa}$ belonging to different paths with index $\kappa \in(i, n], 1 \leq i \leq n$.

The minimum $1 \mathrm{PC} \mathcal{P}_{\mathcal{T}}(H)$ satisfies Property $1 \mathrm{PC}-$ on-H if there is no other minimum $1 \mathrm{PC} \mathcal{P}_{\mathcal{T}}^{\prime}(H)$ having $\varepsilon_{i}^{\prime(n)}$ endpoints $v_{\kappa^{\prime}}$ belonging to different paths with index $\kappa^{\prime} \in(i, n]$ such that $\varepsilon_{i}^{\prime(n)}>\varepsilon_{i}^{(n)}, 1 \leq i<\varrho$, where $\varrho$ is the index of the rightmost endpoint of a path in $\mathcal{P}_{\mathcal{T}}(H)$, and exactly one of the following holds: 
(i) $\varepsilon_{i}^{\prime(n)} \leq \varepsilon_{i}^{(n)}, \varrho \leq i \leq n$;

(ii) $\varepsilon_{i}^{\prime(n)}=\varepsilon_{i}^{(n)}+1, \varrho \leq i<\varrho^{\prime}$ and $\varepsilon_{i}^{\prime(n)}=\varepsilon_{i}^{(n)}, \varrho^{\prime} \leq i \leq n$, where $\varrho^{\prime}$ is the index of the rightmost endpoint of a path in $\mathcal{P}_{\mathcal{T}}^{\prime}(H)$, and there exists a vertex $v_{z}$ such that $\varepsilon_{z}^{(n)}>\varepsilon_{z}^{\prime(n)}$ and there exists no vertex $v_{z^{\prime}}$ such that $\varepsilon_{z^{\prime}}^{\prime(n)}>\varepsilon_{z^{\prime}}^{(n)}, 1 \leq z, z^{\prime}<\varrho$.

Recall that a trivial path has two endpoints that coincide. We next prove Lemmas 4.1-4.6 that concern the operations performed by the Algorithm 1PC_INTERVAL when vertex $v_{n}$ is processed. These lemmas make the proof of Theorem 4.1 easier to follow.

Lemma 4.1 Let $G$ be an interval graph and suppose that Algorithm 1PC_Interval computes a minimum $1 \mathrm{PC} \mathcal{P}_{\mathcal{T}}(G[S])$ of the graph $G[S], S=\left\{v_{1}, v_{2}, \ldots, v_{n-1}\right\}$, on at most $n-1$ vertices satisfying Property $1 \mathrm{PC}-\mathrm{on}-G[S]$. Let $\lambda_{\mathcal{T}}(G[S])$ be the size of $\mathcal{P}_{\mathcal{T}}(G[S])$ and let $v_{n}$ be a non-terminal vertex. If the algorithm uses $v_{n}$ to bridge two paths (operation bridge), then Algorithm 1PC_Interval computes a minimum 1PC $\mathcal{P}_{\mathcal{T}}(G)$ of the graph $G$ satisfying Property 1 PC-on- $G$.

Proof Let $\lambda_{\mathcal{T}}(G)$ be the size of $\mathcal{P}_{\mathcal{T}}(G)$. Clearly, the size $\lambda_{\mathcal{T}}^{\prime}(G)$ of a minimum $1 \mathrm{PC}$ of $G$ is equal to $\lambda_{\mathcal{T}}(G[S])-1$ or $\lambda_{\mathcal{T}}(G[S])$ or $\lambda_{\mathcal{T}}(G[S])+1$. When the algorithm processes vertex $v_{n}$, it uses $v_{n}$ to bridge two paths (operation bridge), that is, $\lambda_{\mathcal{T}}(G)=\lambda_{\mathcal{T}}(G[S])-1$; consequently, $\mathcal{P}_{\mathcal{T}}(G)$ is a minimum $1 \mathrm{PC}$ of $G$, that is, $\lambda_{\mathcal{T}}^{\prime}(G)=\lambda_{\mathcal{T}}(G)$.

Algorithm 1PC_INTERVAL computes a minimum 1PC $\mathcal{P}_{\mathcal{T}}(G[S])$ of the interval graph $G[S], S=\left\{v_{1}, v_{2}, \ldots, v_{n-1}\right\}$, on at most $n-1$ vertices having $\varepsilon_{i}^{(n-1)}$ endpoints $v_{\kappa}$ belonging to different paths with index $\kappa \in(i, n-1], 1 \leq i \leq n-1$, such that there is no other minimum $1 \mathrm{PC} \mathcal{P}_{\mathcal{T}}^{\prime}(G[S])$ having $\varepsilon_{i}^{\prime(n-1)}$ endpoints $v_{\kappa^{\prime}}$ belonging to different paths with index $\kappa^{\prime} \in(i, n-1]$ such that $\varepsilon_{i}^{(n-1)}>\varepsilon_{i}^{(n-1)}, 1 \leq i<d$, where $d$ is the index of the rightmost endpoint of a path in $\mathcal{P}_{\mathcal{T}}(G[S])$, and exactly one of the following holds:

(i) $\varepsilon_{i}^{\prime(n-1)} \leq \varepsilon_{i}^{(n-1)}, d \leq i \leq n-1$;

(ii) $\varepsilon_{i}^{\prime(n-1)}=\varepsilon_{i}^{(n-1)}+1, d \leq i<d^{\prime}$ and $\varepsilon_{i}^{\prime(n-1)}=\varepsilon_{i}^{(n-1)}, d^{\prime} \leq i \leq n-1$, where $d^{\prime}$ is the index of the rightmost endpoint of a path in $\mathcal{P}_{\mathcal{T}}^{\prime}(G[S])$, and there exists a vertex $v_{q}$ such that $\varepsilon_{q}^{(n-1)}>\varepsilon_{q}^{\prime(n-1)}$, and there exists no vertex $v_{q^{\prime}}$ such that $\varepsilon_{q^{\prime}}^{(n-1)}>\varepsilon_{q^{\prime}}^{(n-1)}, 1 \leq q, q^{\prime}<d$.

We distinguish the following cases:

Case 1. Suppose that $\varepsilon_{i}^{\prime(n-1)} \leq \varepsilon_{i}^{(n-1)}, d \leq i \leq n-1$. We show that the algorithm computes a minimum $1 \mathrm{PC} \mathcal{P}_{\mathcal{T}}(G)$ of the graph $G$ having $\varepsilon_{i}^{(n)}$ endpoints $v_{\kappa}$ belonging to different paths with index $\kappa \in(i, n], 1 \leq i \leq n$, such that there is no other minimum 1PC $\mathcal{P}_{\mathcal{T}}^{\prime}(G)$ having $\varepsilon_{i}^{\prime(n)}$ endpoints $v_{\kappa^{\prime}}$ belonging to different paths with index $\kappa^{\prime} \in(i, n]$ such that $\varepsilon_{i}^{\prime(n)}>\varepsilon_{i}^{(n)}, 1 \leq i \leq n$. Clearly, vertex $v_{n}$ is an internal vertex of a path in any other minimum $1 \mathrm{PC} \mathcal{P}_{\mathcal{T}}^{\prime}(G)$, otherwise removing it from $\mathcal{P}_{\mathcal{T}}^{\prime}(G)$ would result to a $1 \mathrm{PC}$ of $G[S]$ of size $\leq \lambda_{\mathcal{T}}(G[S])-1$, a contradiction. Assume that there exists a minimum $1 \mathrm{PC} \mathcal{P}_{\mathcal{T}}^{\prime}(G)$ having an index, say, $k-1$, for which we have 
$\varepsilon_{k-1}^{\prime(n)}$ endpoints $v_{\kappa^{\prime}}$ belonging to different paths with index $\kappa^{\prime} \in(k-1, n]$ such that $\varepsilon_{k-1}^{\prime(n)}>\varepsilon_{k-1}^{(n)}$. Suppose that $\varepsilon_{k-1}^{\prime(n)}-\varepsilon_{k-1}^{(n)}=1$. Note that $\varepsilon_{1}^{(n)} \geq \varepsilon_{1}^{\prime(n)}$. Indeed, let $\mathcal{P}_{\mathcal{T}}^{\prime}(G)$ be a minimum 1PC of $G$ having $\varepsilon_{1}^{\prime(n)}=\varepsilon_{1}^{(n)}+1$ endpoints $v_{\kappa^{\prime}}$ belonging to different paths with index $\kappa^{\prime}>1$. Suppose that vertex $v_{1}$ is an internal vertex in $\mathcal{P}_{\mathcal{T}}(G)$. Then, the algorithm constructs $\varepsilon_{1}^{(n)}$ paths while $\mathcal{P}_{\mathcal{T}}^{\prime}(G)$ contains at least $\varepsilon_{1}^{(n)}+1$ paths, a contradiction. Suppose that vertex $v_{1}$ is an endpoint in $\mathcal{P}_{\mathcal{T}}(G)$. Note that, according to the algorithm, if $v_{1}$ has degree greater or equal to one, then it belongs to a path containing more than one vertex. Consequently, the other endpoint of the path containing $v_{1}$ is one of the $\varepsilon_{1}^{(n)}$ endpoints, and, thus, the algorithm constructs $\varepsilon_{1}^{(n)}$ paths while $\mathcal{P}_{\mathcal{T}}^{\prime}(G)$ contains at least $\varepsilon_{1}^{(n)}+1$ paths, a contradiction. Since $\varepsilon_{1}^{(n)} \geq \varepsilon_{1}^{\prime(n)}$ there exists a vertex $v_{j}, 1 \leq j<k-1$, such that $\varepsilon_{j}^{(n)}=\varepsilon_{j}^{\prime(n)}$. This implies that vertex $v_{j+1}$ is the right endpoint of a path $P$ in the minimum $1 \mathrm{PC} \mathcal{P}_{\mathcal{T}}(G)$ constructed by the algorithm. Without loss of generality, we assume that $\varepsilon_{i}^{\prime(n)}=\varepsilon_{i}^{(n)}+1, j+1 \leq i<k-1$.

Let $P^{\prime}=\left(\ldots, v_{a}, v_{n}, v_{b}, \ldots\right)$ be the path of $\mathcal{P}_{\mathcal{T}}^{\prime}(G)$ containing vertex $v_{n}$. Then, $j+1<a$ and $j+1<b$. Indeed, suppose that at least one of $v_{a}$ and $v_{b}$ has index less or equal to $j+1$, say, $a<j+1$. Since $v_{a} v_{n} \in E(G)$ we have $v_{j+1} v_{n} \in E(G)$. Let $P=\left(\ldots, v_{c}, v_{n}, v_{d}, \ldots\right)$ be the path of $\mathcal{P}_{\mathcal{T}}(G)$ containing vertex $v_{n}$. Suppose that $v_{c}<v_{j+1}$ and $v_{d}<v_{j+1}$. Then, due to the induction hypothesis, both of the endpoints of $P$ have index greater than $j+1$. However, according to the algorithm (operation bridge), such an ordering of the endpoints cannot exist, a contradiction. Suppose that $v_{c}>v_{j+1}$ and $v_{d}>v_{j+1}$. Then, due to the induction hypothesis, at least one of the endpoints of $P$ should have index greater than $j+1$. Again, according to the algorithm (operation bridge), such an ordering of the endpoints cannot exist, a contradiction. Suppose now that $v_{c}<v_{j+1}$ and $v_{d}>v_{j+1}$. Then, due to the induction hypothesis, computing a 1PC of $G[S]$ we had case (e) which is described in Sect. 3 and $v_{j+1}=v_{s}$. However, in this case, vertex $v_{j+1}$ would not be the right endpoint of a path in $\mathcal{P}_{\mathcal{T}}(G[S])$, a contradiction.

Consequently, $j+1<a$ and $j+1<b$. Then, both endpoints of $P$ have indexes less than $j+1$. This implies that vertex $v_{n}$ has bridged two non-terminal paths for which we have an endpoint of one path between the endpoints of the other, which is a contradiction according to Lemma 3.3.

Consequently, we have shown that there does not exist a minimum $1 \mathrm{PC} \mathcal{P}_{\mathcal{T}}^{\prime}(G)$ having an index, say, $k-1$, for which we have $\varepsilon_{k-1}^{\prime(n)}$ endpoints $v_{\mathcal{K}^{\prime}}$ belonging to different paths with index $\kappa^{\prime} \in(k-1, n]$, where $\varepsilon_{k-1}^{\prime(n)}>\varepsilon_{k-1}^{(n)}$.

Case 2. Suppose that $\varepsilon_{i}^{\prime(n-1)}=\varepsilon_{i}^{(n-1)}+1, d \leq i<d^{\prime}$ and $\varepsilon_{i}^{(n-1)}=\varepsilon_{i}^{(n-1)}, d^{\prime} \leq$ $i \leq n-1$, where $d^{\prime}$ is the index of the rightmost endpoint of a path in $\mathcal{P}_{\mathcal{T}}^{\prime}(G[S])$, and there exists a vertex $v_{q}$ such that $\varepsilon_{q}^{(n-1)}>\varepsilon_{q}^{\prime(n-1)}$, and there exists no vertex $v_{q}^{\prime}$ such that $\varepsilon_{q}^{\prime(n-1)}>\varepsilon_{q}^{(n-1)}, 1 \leq q, q^{\prime}<d$. We show that the algorithm computes a minimum $1 \mathrm{PC} \mathcal{P}_{\mathcal{T}}(G)$ of the graph $G$ having $\varepsilon_{i}^{(n)}$ endpoints $v_{\kappa}$ belonging to different paths with index $\kappa \in(i, n], 1 \leq i \leq n$, such that there is no other minimum $1 \mathrm{PC} \mathcal{P}_{\mathcal{T}}^{\prime}(G)$ having $\varepsilon_{i}^{\prime(n)}$ endpoints $v_{\kappa^{\prime}}$ belonging to different paths with index $\kappa^{\prime} \in(i, n], 1 \leq i \leq \varrho$, such that $\varepsilon_{i}^{\prime(n)}>\varepsilon_{i}^{(n)}, 1 \leq i<\varrho$, where $\varrho$ is the index of the rightmost endpoint of a path in $\mathcal{P}_{\mathcal{T}}(G)$, and exactly one of the following holds: 
(i) $\varepsilon_{i}^{\prime(n)} \leq \varepsilon_{i}^{(n)}, \varrho \leq i \leq n$;

(ii) $\varepsilon_{i}^{\prime(n)}=\varepsilon_{i}^{(n)}+1, \varrho \leq i<\varrho^{\prime}$ and $\varepsilon_{i}^{\prime(n)}=\varepsilon_{i}^{(n)}, \varrho^{\prime} \leq i \leq n$, where $\varrho^{\prime}$ is the index of the rightmost endpoint of a path in $\mathcal{P}_{\mathcal{T}}^{\prime}(G)$, and there exists a vertex $v_{z}$ such that $\varepsilon_{z}^{(n)}>\varepsilon_{z}^{\prime(n)}$, and there exists no vertex $v_{z}^{\prime}$ such that $\varepsilon_{z}^{\prime(n)}>\varepsilon_{z}^{(n)}, 1 \leq z, z^{\prime}<\varrho$.

Suppose that there exists a minimum $1 \mathrm{PC} \mathcal{P}_{\mathcal{T}}^{\prime}(G)$ such that $\varepsilon_{\varrho}^{(n)}=\varepsilon_{\varrho}^{\prime(n)}=0$. Then, similarly to Case 1 , we show that there is no other minimum $1 \mathrm{PC} \mathcal{P}_{\mathcal{T}}^{\prime}(G)$ such that $\varepsilon_{i}^{\prime(n)}>\varepsilon_{i}^{(n)}, 1 \leq i<n$.

Suppose now that there exists a minimum $1 \mathrm{PC} \mathcal{P}_{\mathcal{T}}^{\prime}(G)$ such that $\varepsilon_{i}^{\prime(n)}=\varepsilon_{i}^{(n)}+1$, $\varrho \leq i<\varrho^{\prime}$ and $\varepsilon_{i}^{\prime(n)}=\varepsilon_{i}^{(n)}, \varrho^{\prime} \leq i \leq n$, where $\varrho^{\prime}$ is the index of the rightmost endpoint of a path in $\mathcal{P}_{\mathcal{T}}^{\prime}(G)$. We show that there exists a vertex $v_{z}$ such that $\varepsilon_{z}^{(n)}>\varepsilon_{z}^{\prime(n)}$, $1 \leq z<\varrho$ and there exists no vertex $v_{z^{\prime}}, 1 \leq z^{\prime}<\varrho$, such that $\varepsilon_{z^{\prime}}^{(n)}>\varepsilon_{z^{\prime}}^{(n)}$.

Note that, there cannot exist a minimum $1 \mathrm{PC} \mathcal{P}_{\mathcal{T}}^{\prime}(G)$ such that $\varepsilon_{i}^{\prime(n)}=\varepsilon_{i}^{(n)}+2$, $\varrho \leq i<\varrho^{\prime}$. Indeed, suppose that there exists a minimum $1 \mathrm{PC} \mathcal{P}_{\mathcal{T}}^{\prime}(G)$ such that $\varepsilon_{i}^{\prime(n)}=$ $\varepsilon_{i}^{(n)}+2, \varrho \leq i<\varrho^{\prime}$. Consider the case where $v_{n}$ belongs to a path in $\mathcal{P}_{\mathcal{T}}^{\prime}(G)$ and it is connected to vertices $v_{a^{\prime}}$ and $v_{b^{\prime}}$ such that $\varrho<a^{\prime}$ and $\varrho<b^{\prime}$. Then, removing $v_{n}$ from $\mathcal{P}_{\mathcal{T}}^{\prime}(G)$ we obtain a minimum 1 PC of $G[S]$ such that $\varepsilon_{\varrho}^{\prime(n-1)}=\varepsilon_{\varrho}^{\prime(n)}+1=\varepsilon_{\varrho}^{(n)}+3$ or $\varepsilon_{\varrho}^{\prime(n-1)}=\varepsilon_{\varrho}^{\prime(n)}+2=\varepsilon_{\varrho}^{(n)}+4$. If $v_{n}$ belongs to a path in $\mathcal{P}_{\mathcal{T}}(G)$ and it is connected to vertices $v_{a}$ and $v_{b}$, then removing $v_{n}$ from $\mathcal{P}_{\mathcal{T}}(G)$ we obtain a minimum $1 \mathrm{PC}$ of $G[S]$ such that $\varepsilon_{\varrho}^{(n-1)} \leq \varepsilon_{\varrho}^{(n)}+2$. Thus, there exist three paths in $\mathcal{P}_{\mathcal{T}}(G)$ such that there are no left endpoints between their right endpoints in $\pi$, a contradiction. Consider now the case where $v_{n}$ belongs to a path in $\mathcal{P}_{\mathcal{T}}^{\prime}(G)$ and it is connected to vertices $v_{a^{\prime}}$ and $v_{b^{\prime}}$ such that $\varrho<a^{\prime}$ and $\varrho>b^{\prime}$. Then, $v_{n} v_{\varrho} \in E(G)$. Note that $v_{n}$ belongs to a path $P$ in $\mathcal{P}_{\mathcal{T}}(G)$ and it is connected to vertices $v_{a}$ and $v_{b}$ such that $a<\varrho$ and $\varrho<b$. Removing $v_{n}$ from $\mathcal{P}_{\mathcal{T}}(G)$ should result to a minimum 1 PC of $G[S]$ such that $\varepsilon_{\varrho}^{(n-1)}=\varepsilon_{\varrho}^{(n)}+1$. Consequently, both endpoints of $P$ have index less than $\varrho$, which implies that there exists a specific ordering of the endpoints of the paths in $\mathcal{P}_{\mathcal{T}}(G[S])$, which, according to the algorithm, is not possible, a contradiction. The case where $v_{n}$ belongs to a path in $\mathcal{P}_{\mathcal{T}}^{\prime}(G)$ and it is connected to vertices $v_{a^{\prime}}$ and $v_{b^{\prime}}$ such that $\varrho>a^{\prime}$ and $\varrho>b^{\prime}$ is similar.

Using similar arguments with Case 1 , we show that there exists no vertex $v_{z^{\prime}}$, $1 \leq z^{\prime}<\varrho$, such that $\varepsilon_{z^{\prime}}^{\prime(n)}>\varepsilon_{z^{\prime}}^{(n)}$. Suppose that $\varepsilon_{i}^{(n)}=\varepsilon_{i}^{\prime(n)}, 1 \leq i<\varrho$. Note that $v_{n}$ belongs to a path $P=\left(v_{\ell}, \ldots, v_{a}, v_{n}, v_{b}, \ldots, v_{r}\right) \in \mathcal{P}_{\mathcal{T}}(G)$ such that $a>\varrho$ and $b>$ $\varrho$. Thus, $v_{n}$ belongs to a path $P^{\prime}=\left(v_{\ell^{\prime}}, \ldots, v_{a^{\prime}}, v_{n}, v_{b^{\prime}}, \ldots, v_{r^{\prime}}\right) \in \mathcal{P}_{\mathcal{T}}^{\prime}(G)$ such that $a^{\prime}>\varrho$ and $b^{\prime}>\varrho$. If we remove $v_{n}$ from $\mathcal{P}_{\mathcal{T}}^{\prime}(G)$ then there exists a vertex $v_{z}=v_{b^{\prime}-1}$ such that $\varepsilon_{z}^{\prime(n-1)}=\varepsilon_{z}^{\prime(n)}+1$. This implies that $r^{\prime}>b^{\prime}$. Furthermore, if we remove $v_{n}$ from $\mathcal{P}_{\mathcal{T}}(G)$ and we obtain $\varepsilon_{z}^{(n-1)}=\varepsilon_{z}^{(n)}+1$, which implies that $b=b^{\prime}$, then $\varepsilon_{i}^{(n-1)}=\varepsilon_{i}^{\prime(n-1)}, 1 \leq i<\varrho$, a contradiction. If we remove $v_{n}$ from $\mathcal{P}_{\mathcal{T}}^{\prime}(G)$ and we obtain $\varepsilon_{z}^{(n-1)}=\varepsilon_{z}^{(n)}+2$, then there exists a specific ordering of the endpoints of the paths in $\mathcal{P}_{\mathcal{T}}(G[S])$ which, according to the algorithm, is not possible, a contradiction. Consequently, there exists a vertex $v_{z}$ such that $\varepsilon_{z}^{(n)}>\varepsilon_{z}^{\prime(n)}, 1 \leq z<\varrho$. 
Lemma 4.2 Let $G$ be an interval graph and suppose that Algorithm 1PC_Interval computes a minimum $1 \mathrm{PC} \mathcal{P}_{\mathcal{T}}(G[S])$ of the graph $G[S], S=\left\{v_{1}, v_{2}, \ldots, v_{n-1}\right\}$, on at most $n-1$ vertices satisfying Property 1 PC-on- $G[S]$. Let $\lambda_{\mathcal{T}}(G[S])$ be the size of $\mathcal{P}_{\mathcal{T}}(G[S])$ and let $v_{n}$ be a non-terminal vertex. If during the process of vertex $v_{n}$ the algorithm constructs a $1 \mathrm{PC} \mathcal{P}_{\mathcal{T}}\left(G\left[S-\left\{v_{t}\right\}\right]\right)$ of $G\left[S-\left\{v_{t}\right\}\right]$ of size $\lambda_{\mathcal{T}}(G[S])-1$, where $v_{t}$ is the terminal vertex, and then connects the path $\left(v_{t}, v_{n}\right)$ to an existing path $P$, then Algorithm 1PC_Interval computes a minimum $1 \mathrm{PC} \mathcal{P}_{\mathcal{T}}(G)$ of the graph $G$ satisfying Property $1 \mathrm{PC}-\mathrm{on}-G$.

Proof Algorithm 1PC_InTERVAL computes a minimum 1PC $\mathcal{P}_{\mathcal{T}}(G[S])$ of the interval graph $G[S], S=\left\{v_{1}, v_{2}, \ldots, v_{n-1}\right\}$, on at most $n-1$ vertices having $\varepsilon_{i}^{(n-1)}$ endpoints $v_{\kappa}$ belonging to different paths with index $\kappa \in(i, n-1], 1 \leq i \leq n-1$, such that there is no other minimum $1 \mathrm{PC} \mathcal{P}_{\mathcal{T}}^{\prime}(G[S])$ having $\varepsilon_{i}^{\prime(n-1)}$ endpoints $v_{\mathcal{K}^{\prime}}$ belonging to different paths with index $\kappa^{\prime} \in(i, n-1]$ such that $\varepsilon_{i}^{\prime(n-1)}>\varepsilon_{i}^{(n-1)}$, $1 \leq i<d$, where $d$ is the index of the rightmost endpoint of a path in $\mathcal{P}_{\mathcal{T}}(G[S])$, and, moreover, exactly one of the following holds:

(i) $\varepsilon_{i}^{\prime(n-1)} \leq \varepsilon_{i}^{(n-1)}, d \leq i \leq n-1$;

(ii) $\varepsilon_{i}^{\prime(n-1)}=\varepsilon_{i}^{(n-1)}+1, d \leq i<d^{\prime}$ and $\varepsilon_{i}^{\prime(n-1)}=\varepsilon_{i}^{(n-1)}, d^{\prime} \leq i \leq n-1$, where $d^{\prime}$ is the index of the rightmost endpoint of a path in $\mathcal{P}_{\mathcal{T}}^{\prime}(G[S])$, and there exists a vertex $v_{q}$ such that $\varepsilon_{q}^{(n-1)}>\varepsilon_{q}^{\prime(n-1)}$, and there exists no vertex $v_{q^{\prime}}$ such that $\varepsilon_{q^{\prime}}^{(n-1)}>\varepsilon_{q^{\prime}}^{(n-1)}, 1 \leq q, q^{\prime}<d$.

When the algorithm processes vertex $v_{n}$, it constructs a $1 \mathrm{PC} \mathcal{P}_{\mathcal{T}}\left(G\left[S-\left\{v_{t}\right\}\right]\right)$ of $G\left[S-\left\{v_{t}\right\}\right]$ of size $\lambda_{\mathcal{T}}(G[S])-1$, where $v_{t}$ is the terminal vertex, and then connects the path $\left(v_{t}, v_{n}\right)$ to an existing path $P$. This operation is performed when vertex $v_{n}$ sees the endpoints of at least one non-terminal path, say $P_{1}=\left(v_{r}, \ldots, v_{s}\right)$, the terminal vertex $v_{t}$ and no other endpoint of the terminal path, say, $P_{2}=\left(v_{t}, \ldots, v_{\ell}\right)$. Then, the terminal path, $P_{2}$, has the same endpoints as in $\mathcal{P}_{\mathcal{T}}(G[S])$, the vertices of $P_{1}$ become internal vertices of $P_{2}$, while all the other paths in $\mathcal{P}_{\mathcal{T}}(G)$ remain the same as in $\mathcal{P}_{\mathcal{T}}(G[S])$. It is easy to see that there exists a path $P$ in $\mathcal{P}_{\mathcal{T}}\left(G\left[S-\left\{v_{t}\right\}\right]\right)$ having an endpoint, say, $v_{s^{\prime}}$, with index greater than $t$, and thus, $v_{s^{\prime}} \in N\left(v_{n}\right)$. Note that when the connect operation is performed, it may use a vertex of the terminal path in order to increase the value of an $\varepsilon_{i}^{(n-1)}, 1 \leq i<n-1$, and, in this case, $\varepsilon_{d}^{(n-1)}<\varepsilon_{d}^{(n-1)}$. Then, for the endpoints of the terminal path, say, $v_{t} \in \mathcal{T}$ and $v_{\ell}$, we have $v_{t}<v_{\ell}$. Consequently, since vertex $v_{n}$ sees the endpoints of $P_{1}$, the terminal vertex $v_{t}$ and no other endpoint of the terminal path $P_{2}$, if operation connect was called previously, it cannot have used a vertex of the terminal path and, thus, $\varepsilon_{d}^{(n)}<\varepsilon_{d}^{\prime(n)}$ cannot hold. Consequently, $\varepsilon_{i}^{\prime(n-1)} \leq \varepsilon_{i}^{(n-1)}, d \leq i \leq n-1$.

The above procedure results to a $1 \mathrm{PC}$ of $G$ of size $\lambda_{\mathcal{T}}(G)=\lambda_{\mathcal{T}}(G[S])-1$; consequently, $\mathcal{P}_{\mathcal{T}}(G)$ is a minimum $1 \mathrm{PC}$ of $G$, that is, $\lambda_{\mathcal{T}}^{\prime}(G)=\lambda_{\mathcal{T}}(G)$.

We show that the algorithm computes a minimum 1PC $\mathcal{P}_{\mathcal{T}}(G)$ of the graph $G$ having $\varepsilon_{i}^{(n)}$ endpoints $v_{\kappa}$ belonging to different paths with index $\kappa \in(i, n], 1 \leq i \leq n$, such that there is no other minimum $1 \mathrm{PC} \mathcal{P}_{\mathcal{T}}^{\prime}(G)$ having $\varepsilon_{i}^{\prime(n)}$ endpoints $v_{\kappa^{\prime}}$ belonging to different paths with index $\kappa^{\prime} \in(i, n]$ such that $\varepsilon_{i}^{\prime(n)}>\varepsilon_{i}^{(n)}, 1 \leq i \leq n$. Clearly, 
vertex $v_{n}$ is an internal vertex of a path in any other minimum $1 \mathrm{PC} \mathcal{P}_{\mathcal{T}}^{\prime}(G)$, otherwise removing it from $\mathcal{P}_{\mathcal{T}}^{\prime}(G)$ would result to a $1 \mathrm{PC}$ of $G[S]$ of size less or equal to $\lambda_{\mathcal{T}}(G[S])-1$, a contradiction. Suppose that there exists a minimum $1 \mathrm{PC} \mathcal{P}_{\mathcal{T}}^{\prime}(G)$ having an index, say, $k-1$, such that $\varepsilon_{k-1}^{\prime(n)}>\varepsilon_{k-1}^{(n)}$ and $1 \leq k-1<t-1$. This implies that $\varepsilon_{k}^{\prime(n)}=\varepsilon_{k}^{(n)}$ and there exists a vertex $v_{k^{\prime}-1}$ such that $k^{\prime}-1<k-1$ and $\varepsilon_{k^{\prime}-1}^{\prime(n)}=\varepsilon_{k^{\prime}-1}^{(n)}$. Clearly, $v_{k^{\prime}} v_{n} \notin E(G)$. Removing $v_{n}$ from $\mathcal{P}_{\mathcal{T}}^{\prime}(G)$ results to a minimum 1PC of $G[S]$ having at least two non-terminal neighbors of $v_{n}$ as endpoints belonging to different paths, say, $v_{f}$ and $v_{g}$; suppose that at least one of them has index greater than $k$. Then, $\varepsilon_{k-1}^{\prime(n-1)}>\varepsilon_{k-1}^{(n-1)}$, a contradiction. Thus, $v_{k^{\prime}}<v_{f}<v_{k}$ and $v_{k^{\prime}}<v_{g}<v_{k}$ and the right endpoint of the path that they belong, has also index less than $k$. Thus, $\varepsilon_{k^{\prime}}^{\prime(n-1)}=\varepsilon_{k^{\prime}}^{\prime(n)}+1=\varepsilon_{k^{\prime}}^{(n)}+1+1=\varepsilon_{k^{\prime}}^{(n-1)}+1$ or $\varepsilon_{k^{\prime}}^{\prime(n-1)}=\varepsilon_{k^{\prime}}^{\prime(n)}+$ $2=\varepsilon_{k^{\prime}}^{(n)}+1+2=\varepsilon_{k^{\prime}}^{(n-1)}+2$, a contradiction. Suppose now that $t \leq k-1 \leq n$. Since there cannot exist a vertex $v_{\ell}$ such that $1 \leq \ell<t-1$ and $\varepsilon_{\ell}^{\prime(n)}>\varepsilon_{\ell}^{(n)}$, there exists a vertex $v_{k^{\prime}-1}$ such that $k^{\prime}-1<k-1$ and $\varepsilon_{k^{\prime}-1}^{\prime(n)}=\varepsilon_{k^{\prime}-1}^{(n)}$ and $t \leq k^{\prime}$. Clearly, $v_{k^{\prime}} v_{n} \in E(G)$. If $v_{s}$ is the right endpoint of $P_{1}$, then $k-1<s$ and thus $k^{\prime}-1<s$. However, according to the algorithm, there cannot exist an endpoint between vertices $v_{t}$ and $v_{s}$, a contradiction.

Lemma 4.3 Let $G$ be an interval graph and suppose that Algorithm 1PC_Interval computes a minimum $1 \mathrm{PC} \mathcal{P}_{\mathcal{T}}(G[S])$ of the graph $G[S], S=\left\{v_{1}, v_{2}, \ldots, v_{n-1}\right\}$, on at most $n-1$ vertices satisfying Property 1 PC-on- $G[S]$. Let $\lambda_{\mathcal{T}}(G[S])$ be the size of $\mathcal{P}_{\mathcal{T}}(G[S])$ and let $v_{n}$ be a non-terminal vertex. If during the process of vertex $v_{n}$ the algorithm constructs a $1 \mathrm{PC} \mathcal{P}_{\mathcal{T}}\left(G\left[S-\left\{v_{t}\right\}\right]\right)$ of $G\left[S-\left\{v_{t}\right\}\right]$ of size $\lambda_{\mathcal{T}}(G[S])$, where $v_{t}$ is the terminal vertex, connects $v_{t}$ to the leftmost left endpoint it sees, and uses $v_{n}$ to bridge two paths, then Algorithm 1PC_Interval computes a minimum $1 \mathrm{PC} \mathcal{P}_{\mathcal{T}}(G)$ of the graph $G$ satisfying Property 1 PC-on-G.

Proof Algorithm 1PC_INTERVAL computes a minimum 1PC $\mathcal{P}_{\mathcal{T}}(G[S])$ of the interval graph $G[S], S=\left\{v_{1}, v_{2}, \ldots, v_{n-1}\right\}$, on at most $n-1$ vertices having $\varepsilon_{i}^{(n-1)}$ endpoints $v_{\kappa}$ belonging to different paths with index $\kappa \in(i, n-1], 1 \leq i \leq n-1$, such that there is no other minimum $1 \mathrm{PC} \mathcal{P}_{\mathcal{T}}^{\prime}(G[S])$ having $\varepsilon_{i}^{\prime(n-1)}$ endpoints $v_{\kappa^{\prime}}$ belonging to different paths with index $\kappa^{\prime} \in(i, n-1]$ such that $\varepsilon_{i}^{\prime(n-1)}>\varepsilon_{i}^{(n-1)}$, $1 \leq i<d$, where $d$ is the index of the rightmost endpoint of a path in $\mathcal{P}_{\mathcal{T}}(G[S])$, and, moreover, one of the following holds:

(i) $\varepsilon_{i}^{\prime(n-1)} \leq \varepsilon_{i}^{(n-1)}, d \leq i \leq n-1$;

(ii) $\varepsilon_{i}^{\prime(n-1)}=\varepsilon_{i}^{(n-1)}+1, d \leq i<d^{\prime}$ and $\varepsilon_{i}^{\prime(n-1)}=\varepsilon_{i}^{(n-1)}, d^{\prime} \leq i \leq n-1$, where $d^{\prime}$ is the index of the rightmost endpoint of a path in $\mathcal{P}_{\mathcal{T}}^{\prime}(G[S])$, and there exists a vertex $v_{q}$ such that $\varepsilon_{q}^{(n-1)}>\varepsilon_{q}^{\prime(n-1)}$, and there exists no vertex $v_{q^{\prime}}$ such that $\varepsilon_{q^{\prime}}^{\prime(n-1)}>\varepsilon_{q^{\prime}}^{(n-1)}, 1 \leq q, q^{\prime}<d$.

When the algorithm processes vertex $v_{n}$, it constructs a $1 \mathrm{PC} \mathcal{P}_{\mathcal{T}}\left(G\left[S-\left\{v_{t}\right\}\right]\right)$ of $G\left[S-\left\{v_{t}\right\}\right]$ of size $\lambda_{\mathcal{T}}(G[S])$, where $v_{t}$ is the terminal vertex, it connects $v_{t}$ to the leftmost left endpoint it sees, and it uses $v_{n}$ to bridge two paths. This operation is performed when vertex $v_{n}$ sees the endpoints of at least one non-terminal path, say $P_{1}=$ 
$\left(v_{r}, \ldots, v_{s}\right)$, the terminal vertex $v_{t}$ of the terminal path, say, $P_{2}=\left(v_{t}, v_{j}, \ldots, v_{\ell}\right)$ and an internal vertex $v_{j}$ of $P_{2}$, and it does not see $v_{\ell}$. Then, the terminal path, $P_{2}$, has the same endpoints as in $\mathcal{P}_{\mathcal{T}}(G[S])$, the vertices of $P_{1}$ become internal vertices of $P_{2}$, while all the other paths remain the same. Recall that, when the connect operation is performed, it may use a vertex of the terminal path in order to increase the value of an $\varepsilon_{i}^{(n-1)}, 1 \leq i<n-1$, and, in this case, $\varepsilon_{d}^{(n-1)}<\varepsilon_{d}^{(n-1)}$. Then, for the endpoints of the terminal path, say, $v_{t} \in \mathcal{T}$ and $v_{\ell}$, we have $v_{t}<v_{\ell}$. Consequently, since vertex $v_{n}$ sees the endpoints of $P_{1}$, the terminal vertex $v_{t}$ and not $v_{\ell}$, if operation connect was called previously, it cannot have used a vertex of the terminal path and, thus, $\varepsilon_{d}^{(n-1)}<\varepsilon_{d}^{(n-1)}$ cannot hold. Consequently, $\varepsilon_{i}^{(n-1)} \leq \varepsilon_{i}^{(n-1)}, d \leq i \leq n-1$.

Consider the case where vertex $v_{n}$ sees the endpoints of only one non-terminal path, that is, of $P_{1}$. If applying the algorithm to $G\left[S-\left\{v_{t}\right\}\right]$ results to a $1 \mathrm{PC}$ of size $\lambda_{\mathcal{T}}(G[S])-1$ then it contains a path with one endpoint $v_{k}$ such that $t<k$. Indeed, suppose that there does not exist such a path and $P_{1}$ consists of more than one vertex. This implies that all vertices of $P_{1}$ have bridged two paths and therefore we obtain a $1 \mathrm{PC}$ of size less than $\lambda_{\mathcal{T}}(G[S])-1$, a contradiction. Suppose now that the algorithm does not construct a path in the 1PC of $G\left[S-\left\{v_{t}\right\}\right]$ with one endpoint $v_{k}$ such that $t<k$ and let the path $P_{1}$ consist of one vertex, say, $v_{k^{\prime}}$. This implies that vertex $v_{k^{\prime}}$ has bridged two paths and the same holds for every vertex $v_{i}, t \leq i \leq n-1$. Thus, if $v_{k^{\prime}}$ is removed from $G\left[S-\left\{v_{t}\right\}\right]$, the algorithm would construct a minimum $1 \mathrm{PC}$ of size $\lambda_{\mathcal{T}}(G[S])$. Since the size of $\mathcal{P}_{\mathcal{T}}(G[S])$ constructed by the algorithm is $\lambda_{\mathcal{T}}(G[S])$, removing $v_{t}$ and $v_{k^{\prime}}$ results to a $1 \mathrm{PC}$ of size $\lambda_{\mathcal{T}}(G[S])-1$. Consequently, $v_{k^{\prime}}$ cannot be used for bridging two paths in $\mathcal{P}_{\mathcal{T}}\left(G\left[S-\left\{v_{t}\right\}\right]\right)$. This implies that the 1PC of $G\left[S-\left\{v_{t}\right\}\right]$ constructed by the algorithm contains a path with an endpoint $v_{k}$ such that $t<k$. It is easy to see that if vertex $v_{n}$ sees the endpoints of more than one non-terminal path, applying the algorithm to $G\left[S-\left\{v_{t}\right\}\right]$ results to a 1PC of size $\lambda_{\mathcal{T}}(G[S])-1$ having a path with one endpoint $v_{k}$ such that $t<k$.

The above procedure results to a $1 \mathrm{PC}$ of $G$ of size $\lambda_{\mathcal{T}}(G)=\lambda_{\mathcal{T}}(G[S])-1$; consequently, $\mathcal{P}_{\mathcal{T}}(G)$ is a minimum $1 \mathrm{PC}$ of $G$, that is, $\lambda_{\mathcal{T}}^{\prime}(G)=\lambda_{\mathcal{T}}(G)$.

Using similar arguments as in the proof of Lemma 4.2, we show that the algorithm computes a minimum $1 \mathrm{PC} \mathcal{P}_{\mathcal{T}}(G)$ of the graph $G$ having $\varepsilon_{i}^{(n)}$ endpoints $v_{\kappa}$ belonging to different paths with index $\kappa \in(i, n], 1 \leq i \leq n$, such that there is no other minimum $1 \mathrm{PC} \mathcal{P}_{\mathcal{T}}^{\prime}(G)$ having $\varepsilon_{i}^{\prime(n)}$ endpoints $v_{\kappa^{\prime}}$ belonging to different paths with index $\kappa^{\prime} \in$ $(i, n]$ such that $\varepsilon_{i}^{\prime(n)}>\varepsilon_{i}^{(n)}, 1 \leq i \leq n$.

Lemma 4.4 Let $G$ be an interval graph and suppose that Algorithm 1PC_Interval computes a minimum $1 \mathrm{PC} \mathcal{P}_{\mathcal{T}}(G[S])$ of the graph $G[S], S=\left\{v_{1}, v_{2}, \ldots, v_{n-1}\right\}$, on at most $n-1$ vertices satisfying Property 1 PC-on- $G[S]$. Let $\lambda_{\mathcal{T}}(G[S])$ be the size of $\mathcal{P}_{\mathcal{T}}(G[S])$ and let $v_{n}$ be a non-terminal vertex. If during the process of vertex $v_{n}$ the algorithm connects $v_{n}$ to a path or inserts $v_{n}$ into a path, then Algorithm 1PC_Interval computes a minimum $1 \mathrm{PC} \mathcal{P}_{\mathcal{T}}(G)$ of the graph $G$ satisfying Property 1 PC-on- $G$.

Proof Algorithm 1PC_INTERVAL computes a minimum 1PC $\mathcal{P}_{\mathcal{T}}(G[S])$ of the interval graph $G[S], S=\left\{v_{1}, v_{2}, \ldots, v_{n-1}\right\}$, on at most $n-1$ vertices having $\varepsilon_{i}^{(n-1)}$ 
endpoints $v_{\kappa}$ belonging to different paths with index $\kappa \in(i, n-1], 1 \leq i \leq n-1$, such that there is no other minimum $1 \mathrm{PC} \mathcal{P}_{\mathcal{T}}^{\prime}(G[S])$ having $\varepsilon_{i}^{\prime(n-1)}$ endpoints $v_{\kappa^{\prime}}$ belonging to different paths with index $\kappa^{\prime} \in(i, n-1]$ such that $\varepsilon_{i}^{\prime(n-1)}>\varepsilon_{i}^{(n-1)}$, $1 \leq i<d$, where $d$ is the index of the rightmost endpoint of a path in $\mathcal{P}_{\mathcal{T}}(G[S])$, and, moreover, exactly one of the following holds:

(i) $\varepsilon_{i}^{\prime(n-1)} \leq \varepsilon_{i}^{(n-1)}, d \leq i \leq n-1$;

(ii) $\varepsilon_{i}^{\prime(n-1)}=\varepsilon_{i}^{(n-1)}+1, d \leq i<d^{\prime}$ and $\varepsilon_{i}^{\prime(n-1)}=\varepsilon_{i}^{(n-1)}, d^{\prime} \leq i \leq n-1$, where $d^{\prime}$ is the index of the rightmost endpoint of a path in $\mathcal{P}_{\mathcal{T}}^{\prime}(G[S])$, and there exists a vertex $v_{q}$ such that $\varepsilon_{q}^{(n-1)}>\varepsilon_{q}^{\prime(n-1)}$, and there exists no vertex $v_{q^{\prime}}$ such that $\varepsilon_{q^{\prime}}^{(n-1)}>\varepsilon_{q^{\prime}}^{(n-1)}, 1 \leq q, q^{\prime}<d$.

We distinguish the following cases, according to the operation performed by the algorithm during the process of vertex $v_{n}$.

Case A When the algorithm processes vertex $v_{n}$, it connects $v_{n}$ to a path, that is, $\lambda_{\mathcal{T}}(G)=\lambda_{\mathcal{T}}(G[S])$. Suppose that there exists a $1 \mathrm{PC} \mathcal{P}_{\mathcal{T}}^{\prime}(G)$ of size $\lambda_{\mathcal{T}}(G[S])-1$, that is, vertex $v_{n}$ is an internal vertex of a path $P$ in $\mathcal{P}_{\mathcal{T}}^{\prime}(G)$. We distinguish the following cases:

(i) $P=\left(v_{k}, \ldots, v_{r}, v_{n}, v_{s}, \ldots, v_{\ell}\right)$. Removing $v_{n}$ from $P$ results to a minimum 1PC of $G[S]$ having two (non-terminal) neighbors of $v_{n}$ as endpoints belonging to different paths. Since the algorithm does not use $v_{n}$ to bridge two paths, the constructed minimum 1PC of $G[S]$ does not have two (non-terminal) neighbors of $v_{n}$ as endpoints belonging to different paths. Consequently, there is a minimum 1PC of $G[S]$ for which there exists an index $i$ such that $\varepsilon_{i}^{\prime(n)}>\varepsilon_{i}^{(n)}$, a contradiction.

(ii) $P=\left(v_{t}, v_{n}, \ldots, v_{b}\right)$, where $v_{t}$ is the terminal vertex. Removing $v_{n}$ and $v_{t}$ from $P$ results to a minimum $1 \mathrm{PC}$ of $G[S]$ of size $\lambda_{\mathcal{T}}(G[S])-1$, a contradiction. Indeed, since the algorithm does not use $v_{n}$ to bridge two paths, removing $v_{t}$ from $G[S]$ results to $\lambda_{\mathcal{T}}(G[S])$ paths.

Consequently, there does not exist a $1 \mathrm{PC} \mathcal{P}_{\mathcal{T}}^{\prime}(G)$ of size $\lambda_{\mathcal{T}}(G[S])-1$, and, thus, the $1 \mathrm{PC}$ constructed by the algorithm is minimum.

Case A.1. Suppose that $\varepsilon_{i}^{\prime(n-1)} \leq \varepsilon_{i}^{(n-1)}, d \leq i \leq n-1$. We show that the algorithm computes a minimum $1 \mathrm{PC} \mathcal{P}_{\mathcal{T}}(G)$ of the graph $G$ having $\varepsilon_{i}^{(n)}$ endpoints $v_{\kappa}$ belonging to different paths with index $\kappa \in(i, n], 1 \leq i \leq n$, such that there is no other minimum $1 \mathrm{PC} \mathcal{P}_{\mathcal{T}}^{\prime}(G)$ having $\varepsilon_{i}^{\prime(n)}$ endpoints $v_{\kappa^{\prime}}$ belonging to different paths with index $\kappa^{\prime} \in(i, n]$ such that $\varepsilon_{i}^{\prime(n)}>\varepsilon_{i}^{(n)}, 1 \leq i<\varrho$, and exactly one of the following holds:

(i) $\varepsilon_{i}^{\prime(n)} \leq \varepsilon_{i}^{(n)}, \varrho \leq i \leq n$;

(ii) $\varepsilon_{i}^{\prime(n)}=\varepsilon_{i}^{(n)}+1, \varrho \leq i<\varrho^{\prime}$ and $\varepsilon_{i}^{\prime(n)}=\varepsilon_{i}^{(n)}, \varrho^{\prime} \leq i \leq n$, and there exists a vertex $v_{z}$ such that $\varepsilon_{z}^{(n)}>\varepsilon_{z}^{\prime(n)}$, and there exists no vertex $v_{z^{\prime}}$ such that $\varepsilon_{z^{\prime}}^{\prime(n)}>\varepsilon_{z^{\prime}}^{(n)}$, $1 \leq z, z^{\prime}<\varrho$.

Suppose that $v_{n}$ is not an endpoint in $\mathcal{P}_{\mathcal{T}}(G)$; let $P=\left(\ldots, v_{a}, v_{n}, v_{b}, \ldots\right)$. According to operation connect, we break the terminal path of $\mathcal{P}_{\mathcal{T}}(G[S])$, which has 
the terminal vertex, $v_{t}$, as its right endpoint. Note that the terminal vertex is the second rightmost endpoint in $\mathcal{P}_{\mathcal{T}}(G[S]$ ) (see Sect. 3). The second rightmost endpoint of $\mathcal{P}_{\mathcal{T}}(G)$ has index greater than $t$, and $v_{t}$ becomes a left endpoint of a path in $\mathcal{P}_{\mathcal{T}}(G)$. Assume that there exists a minimum $1 \mathrm{PC} \mathcal{P}_{\mathcal{T}}^{\prime}(G)$ having an index, say, $k-1$, for which we have $\varepsilon_{k-1}^{\prime(n)}$ endpoints $v_{\kappa^{\prime}}$ belonging to different paths with index $\kappa^{\prime} \in(k-1, n]$, where $\varepsilon_{k-1}^{\prime(n)}>\varepsilon_{k-1}^{(n)}$. Similarly, to Case 1 of Lemma 4.1, there exists a vertex $v_{j}, 1 \leq j<k-1$, such that $\varepsilon_{j}^{(n)}=\varepsilon_{j}^{\prime(n)}$.

Suppose that $v_{n}$ is an endpoint of a path $P^{\prime}=\left(v_{n}, v_{a^{\prime}}, \ldots\right) \in \mathcal{P}_{\mathcal{T}}^{\prime}(G)$. Then, since $\varrho^{\prime}=n, \varepsilon_{i}^{\prime(n)}=\varepsilon_{i}^{(n)}+1, \varrho \leq i \leq n$. Note that, there cannot exist a minimum $1 \mathrm{PC} \mathcal{P}_{\mathcal{T}}^{\prime}(G)$ such that $\varepsilon_{i}^{\prime(n)}=\varepsilon_{i}^{(n)}+2, \varrho \leq i \leq n$. Indeed, suppose that there exists a minimum $1 \mathrm{PC} \mathcal{P}_{\mathcal{T}}^{\prime}(G)$ such that $\varepsilon_{i}^{\prime(n)}=\varepsilon_{i}^{(n)}+2, \varrho \leq i \leq n$. If we remove $v_{n}$ from $\mathcal{P}_{\mathcal{T}}^{\prime}(G)$ we obtain $\varepsilon_{\varrho}^{\prime(n-1)}=\varepsilon_{\varrho}^{\prime(n)}$ or $\varepsilon_{\varrho}^{\prime(n-1)}=\varepsilon_{\varrho}^{\prime(n)}-1$. However, $\varepsilon_{\varrho}^{\prime(n)}=\varepsilon_{\varrho}^{(n)}+2$ and $\varepsilon_{\varrho}^{(n)}=\varepsilon_{\varrho}^{(n-1)}=0$, a contradiction. According to the connect operation, $v_{n} v_{j+1} \notin E(G)$, thus $v_{a^{\prime}}>v_{j+1}$. If we remove $v_{n}$ from $\mathcal{P}_{\mathcal{T}}^{\prime}(G)$ we obtain $\varepsilon_{j+1}^{\prime(n-1)}=\varepsilon_{j+1}^{\prime(n)}=\varepsilon_{j+1}^{(n)}+1$. However, $\varepsilon_{j+1}^{(n)}=\varepsilon_{j+1}^{(n-1)}$ or $\varepsilon_{j+1}^{(n)}=\varepsilon_{j+1}^{(n-1)}+1$, a contradiction. Consequently, there exists no vertex $v_{z^{\prime}}, 1 \leq z^{\prime}<\varrho$, such that $\varepsilon_{z^{\prime}}^{(n)}>\varepsilon_{z^{\prime}}^{(n)}$. Suppose that $\varepsilon_{i}^{\prime(n)}=\varepsilon_{i}^{(n)}, 1 \leq i<\varrho$. Let $v_{r}$ be the new endpoint created by the connect operation. Again, since $v_{n} v_{r-1} \notin E(G), v_{a^{\prime}}>v_{r-1}$ and if we remove $v_{n}$ from $\mathcal{P}_{\mathcal{T}}^{\prime}(G)$ we obtain $\varepsilon_{r-1}^{\prime(n-1)}=\varepsilon_{r-1}^{\prime(n)}=\varepsilon_{r-1}^{(n)}$. However, $\varepsilon_{r-1}^{(n)}=\varepsilon_{r-1}^{(n-1)}+1$, a contradiction. Consequently, if $\varepsilon_{i}^{\prime(n)}=\varepsilon_{i}^{(n)}+1, \varrho \leq i<\varrho^{\prime}$ and $\varepsilon_{i}^{\prime(n)}=\varepsilon_{i}^{(n)}, \varrho^{\prime} \leq i \leq n$ then there exists a vertex $v_{z}$ such that $\varepsilon_{z}^{(n)}>\varepsilon_{z}^{\prime(n)}, 1 \leq z<\varrho$ and there exists no vertex $v_{z^{\prime}}, 1 \leq z^{\prime}<\varrho$, such that $\varepsilon_{z^{\prime}}^{(n)}>\varepsilon_{z^{\prime}}^{(n)}$. Suppose that the second rightmost endpoint in $\mathcal{P}_{\mathcal{T}}(G)$, say, $v_{f}$, has index less than the second rightmost endpoint in $\mathcal{P}_{\mathcal{T}}^{\prime}(G)$, say, $v_{f^{\prime}}$, that is, $v_{f}<v_{f^{\prime}}$. Then, $\varepsilon_{f-1}^{(n-1)}=\varepsilon_{f-1}^{(n)}-1=\varepsilon_{f-1}^{\prime(n)}-2$ and $\varepsilon_{f-1}^{\prime(n-1)}=\varepsilon_{f-1}^{\prime(n)}-1$, a contradiction.

Suppose that $v_{n}$ is not an endpoint in $\mathcal{P}_{\mathcal{T}}^{\prime}(G)$; let $P^{\prime}=\left(\ldots, v_{a^{\prime}}, v_{n}, v_{b^{\prime}}, \ldots\right)$. Clearly, one of $v_{a^{\prime}}, v_{b^{\prime}}$ is a vertex that could not be an endpoint in $\mathcal{P}_{\mathcal{T}}^{\prime}(G)$. We show that $\varrho^{\prime} \leq \varrho$. Suppose that $\varrho^{\prime}>\varrho$. Since $\varepsilon_{\varrho}^{(n-1)}=\varepsilon_{\varrho}^{(n)}=\varepsilon_{\varrho}^{\prime(n)}-1=0$, then we have $\varepsilon_{\varrho}^{\prime(n-1)}=\varepsilon_{\varrho}^{\prime(n)}-1$, which implies that the new endpoint in $\mathcal{P}_{\mathcal{T}}^{\prime}(G)$ has index greater than the new endpoint created in $\mathcal{P}_{\mathcal{T}}(G)$, a contradiction. It is easy to see that there cannot exist a minimum $1 \mathrm{PC} \mathcal{P}_{\mathcal{T}}^{\prime}(G)$ having an index, say, $k-1$, for which we have $\varepsilon_{k-1}^{\prime(n)}$ endpoints $v_{\kappa^{\prime}}$ belonging to different paths with index $\kappa^{\prime} \in(k-1, n]$ such that $\varepsilon_{k-1}^{\prime(n)}=\varepsilon_{k-1}^{\prime(n-1)}+2$. Let $v_{t}$ be the terminal vertex. We have $\varepsilon_{t-1}^{(n)}=\varepsilon_{t-1}^{\prime(n-1)}$. It is easy to see that there cannot exist a minimum $1 \mathrm{PC} \mathcal{P}_{\mathcal{T}}^{\prime}(G)$ having an index, say, $k-1$, $k-1 \leq t-1$, for which we have $\varepsilon_{k-1}^{\prime(n)}$ endpoints $v_{\kappa^{\prime}}$ belonging to different paths with index $\kappa^{\prime} \in(k-1, n]$, such that $\varepsilon_{k-1}^{\prime(n)}>\varepsilon_{k-1}^{(n)}$.

Suppose that $v_{n}$ is the right endpoint of a path $P=\left(v_{n}, v_{a}, \ldots\right) \in \mathcal{P}_{\mathcal{T}}(G)$. Then, there cannot exist a minimum $1 \mathrm{PC} \mathcal{P}_{\mathcal{T}}^{\prime}(G)$ such that $1=\varepsilon_{\varrho-1}^{(n)}<\varepsilon_{\varrho-1}^{\prime(n)}$. We show that the algorithm computes a minimum $1 \mathrm{PC} \mathcal{P}_{\mathcal{T}}(G)$ of the graph $G$ having $\varepsilon_{i}^{(n)}$ endpoints $v_{\kappa}$ belonging to different paths with index $\kappa \in(i, n], 1 \leq i \leq n$, such that there 
is no other minimum $1 \mathrm{PC} \mathcal{P}_{\mathcal{T}}^{\prime}(G)$ having $\varepsilon_{i}^{\prime(n)}$ endpoints $v_{\mathcal{K}^{\prime}}$ belonging to different paths with index $\kappa^{\prime} \in(i, n]$ such that $\varepsilon_{i}^{\prime(n)}>\varepsilon_{i}^{(n)}, 1 \leq i \leq n$. Assume that there exists a minimum $1 \mathrm{PC} \mathcal{P}_{\mathcal{T}}^{\prime}(G)$ having an index, say, $k-1$, for which we have $\varepsilon_{k-1}^{\prime(n)}$ endpoints $v_{\kappa^{\prime}}$ belonging to different paths with index $\kappa^{\prime} \in(k-1, n]$, where $\varepsilon_{k-1}^{\prime(n)}>\varepsilon_{k-1}^{(n)}$. Similarly, to Case 1 of Lemma 4.1, there exists a vertex $v_{j}, 1 \leq j<k-1$, such that $\varepsilon_{j}^{(n)}=\varepsilon_{j}^{\prime(n)}$.

Suppose that $v_{n}$ is an endpoint of a path $P^{\prime}=\left(v_{n}, v_{a^{\prime}}, \ldots\right) \in \mathcal{P}_{\mathcal{T}}^{\prime}(G)$. Note that if $v_{a^{\prime}}<v_{j+1}$, then $v_{j+1} v_{n} \in E(G)$ and $v_{k}$ should be the terminal vertex belonging to a non-trivial path, otherwise $\mathcal{P}_{\mathcal{T}}(G)$ would not be minimum. Thus, if $v_{j+1}$ is not the terminal vertex, $v_{a}<v_{j+1}$ or $v_{a}=v_{j+1}$. Then, if we remove $v_{n}$ from $\mathcal{P}_{\mathcal{T}}^{\prime}(G)$ and $\mathcal{P}_{\mathcal{T}}(G)$, we obtain $\varepsilon_{j+1}^{(n-1)}=\varepsilon_{j+1}^{(n)}-1=\varepsilon_{j+1}^{\prime(n)}-2$ and $\varepsilon_{j+1}^{\prime(n-1)}=\varepsilon_{j+1}^{\prime(n)}-1$; thus, $\varepsilon_{j+1}^{(n-1)}=\varepsilon_{j+1}^{\prime(n-1)}-1$, a contradiction. On the other hand, if $v_{a^{\prime}}>v_{j+1}$, then, if we remove $v_{n}$ from $\mathcal{P}_{\mathcal{T}}(G)$ and $\mathcal{P}_{\mathcal{T}}^{\prime}(G)$, we obtain $\varepsilon_{j+1}^{(n-1)}=\varepsilon_{j+1}^{(n)}-1=\varepsilon_{j+1}^{\prime(n)}-2$ or $\varepsilon_{j+1}^{(n-1)}=\varepsilon_{j+1}^{(n)}=\varepsilon_{j+1}^{\prime(n)}-1$. Also, $\varepsilon_{j+1}^{\prime(n-1)}=\varepsilon_{j+1}^{\prime(n)}$; thus, $\varepsilon_{j+1}^{(n-1)}=\varepsilon_{j+1}^{\prime(n-1)}-1$, a contradiction.

Suppose that $v_{n}$ is not an endpoint in $\mathcal{P}_{\mathcal{T}}^{\prime}(G)$; let $P^{\prime}=\left(\ldots, v_{a^{\prime}}, v_{n}, v_{b^{\prime}}, \ldots\right)$. If $v_{a^{\prime}} v_{b^{\prime}} \in E(G)$ then if we remove $v_{n}$ from $\mathcal{P}_{\mathcal{T}}^{\prime}(G)$ and $\mathcal{P}_{\mathcal{T}}^{\prime}(G)$, we obtain $\varepsilon_{j+1}^{(n-1)}=$ $\varepsilon_{j+1}^{(n)}-1=\varepsilon_{j+1}^{\prime(n)}-2$ or $\varepsilon_{j+1}^{(n-1)}=\varepsilon_{j+1}^{(n)}=\varepsilon_{j+1}^{\prime(n)}-1$. Also, $\varepsilon_{j+1}^{\prime(n-1)}=\varepsilon_{j+1}^{\prime(n)}$; thus, $\varepsilon_{j+1}^{(n-1)}=\varepsilon_{j+1}^{\prime(n-1)}-1$, a contradiction. Consequently, $v_{a^{\prime}} v_{b^{\prime}} \notin E(G)$; however, we have shown that $v_{n}$ becomes an endpoint in $\mathcal{P}_{\mathcal{T}}(G)$ only when a new right endpoint cannot be created by making $v_{n}$ an internal vertex, a contradiction.

Case A.2. Suppose that $\varepsilon_{i}^{\prime(n-1)}=\varepsilon_{i}^{(n-1)}+1, d \leq i<d^{\prime}$ and $\varepsilon_{i}^{\prime(n-1)}=\varepsilon_{i}^{(n-1)}$, $d^{\prime} \leq i \leq n-1$ and there exists a vertex $v_{q}$ such that $\varepsilon_{q}^{(n-1)}>\varepsilon_{q}^{\prime(n-1)}, 1 \leq q<d$ and there exists no vertex $v_{q^{\prime}}, 1 \leq q^{\prime}<d$, such that $\varepsilon_{q^{\prime}}^{(n-1)}>\varepsilon_{q^{\prime}}^{(n-1)}$. We show that the algorithm computes a minimum $1 \mathrm{PC} \mathcal{P}_{\mathcal{T}}(G)$ of the graph $G$ having $\varepsilon_{i}^{(n)}$ endpoints $v_{\kappa}$ belonging to different paths with index $\kappa \in(i, n], 1 \leq i \leq n$, such that there is no other minimum $1 \mathrm{PC} \mathcal{P}_{\mathcal{T}}^{\prime}(G)$ having $\varepsilon_{i}^{\prime(n)}$ endpoints $v_{\kappa^{\prime}}$ belonging to different paths with index $\kappa^{\prime} \in(i, n]$ such that $\varepsilon_{i}^{\prime(n)}>\varepsilon_{i}^{(n)}, 1 \leq i \leq n$.

Assume that there exists a minimum $1 \mathrm{PC} \mathcal{P}_{\mathcal{T}}^{\prime}(G)$ having an index, say, $k-1$, for which we have $\varepsilon_{k-1}^{\prime(n)}$ endpoints $v_{\kappa^{\prime}}$ belonging to different paths with index $\kappa^{\prime} \in$ $(k-1, n]$, where $\varepsilon_{k-1}^{\prime(n)}>\varepsilon_{k-1}^{(n)}$. Similarly, to Case 1 of Lemma 4.1, there exists a vertex $v_{j}, 1 \leq j<k-1$, such that $\varepsilon_{j}^{(n)}=\varepsilon_{j}^{\prime(n)}$. Using similar arguments as in Case A.1, we show that $v_{n}$ is an endpoint of a path $P^{\prime}=\left(v_{n}, v_{a^{\prime}}, \ldots\right) \in \mathcal{P}_{\mathcal{T}}^{\prime}(G)$ and that there cannot exist a minimum $1 \mathrm{PC} \mathcal{P}_{\mathcal{T}}^{\prime}(G)$ having an index, say, $k-1$, for which we have $\varepsilon_{k-1}^{\prime(n)}$ endpoints $v_{\kappa^{\prime}}$ belonging to different paths with index $\kappa^{\prime} \in(k-1, n]$, where $\varepsilon_{k-1}^{\prime(n)}>\varepsilon_{k-1}^{(n)}$.

Case B When the algorithm processes vertex $v_{n}$, it inserts $v_{n}$ into a path, that is, $\lambda_{\mathcal{T}}(G)=\lambda_{\mathcal{T}}(G[S])$. This implies that $\forall i \geq d$ we have $\varepsilon_{i}^{(n-1)} \leq 1$. Suppose that there exists a $1 \mathrm{PC} \mathcal{P}_{\mathcal{T}}^{\prime}(G)$ of size $\lambda_{\mathcal{T}}(G[S])-1$, that is, vertex $v_{n}$ is an internal vertex of 
a path $P$ in $\mathcal{P}_{\mathcal{T}}^{\prime}(G)$. Then, removing vertex $v_{n}$ from $\mathcal{P}_{\mathcal{T}}^{\prime}(G)$ results to a $1 \mathrm{PC}$ of $G[S]$ of size $\lambda_{\mathcal{T}}(G[S])$, and, thus, minimum, such that there exists an index $i, i \geq d$, for which $\varepsilon_{i}^{\prime(n-1)}=2$, a contradiction.

Consequently, there does not exist a $1 \mathrm{PC} \mathcal{P}_{\mathcal{T}}^{\prime}(G)$ of size $\lambda_{\mathcal{T}}(G[S])-1$, and, thus, the $1 \mathrm{PC}$ constructed by the algorithm is minimum.

Case B.1. Suppose that $\varepsilon_{i}^{\prime(n-1)} \leq \varepsilon_{i}^{(n-1)}, d \leq i \leq n-1$. We show that the algorithm computes a minimum $1 \mathrm{PC} \mathcal{P}_{\mathcal{T}}(G)$ of the graph $G$ having $\varepsilon_{i}^{(n)}$ endpoints $v_{\kappa}$ belonging to different paths with index $\kappa \in(i, n], 1 \leq i \leq n$, such that there is no other minimum $1 \mathrm{PC} \mathcal{P}_{\mathcal{T}}^{\prime}(G)$ having $\varepsilon_{i}^{\prime(n)}$ endpoints $v_{\mathcal{K}^{\prime}}$ belonging to different paths with index $\kappa^{\prime} \in(i, n]$ such that $\varepsilon_{i}^{\prime(n)}>\varepsilon_{i}^{(n)}, 1 \leq i \leq n$.

Assume that there exists a minimum $1 \mathrm{PC} \mathcal{P}_{\mathcal{T}}^{\prime}(G)$ having an index, say, $k-1$, for which we have $\varepsilon_{k-1}^{\prime(n)}$ endpoints $v_{\kappa^{\prime}}$ belonging to different paths with index $\kappa^{\prime} \in$ $(k-1, n]$, where $\varepsilon_{k-1}^{\prime(n)}>\varepsilon_{k-1}^{(n)}$. Similarly, to Case 1 of Lemma 4.1, there exists a vertex $v_{j}, 1 \leq j<k-1$, such that $\varepsilon_{j}^{(n)}=\varepsilon_{j}^{\prime(n)}$.

Suppose that $v_{n}$ is an endpoint of a path $P^{\prime}=\left(v_{n}, v_{t}\right) \in \mathcal{P}_{\mathcal{T}}^{\prime}(G)$, such that $v_{t} \in \mathcal{T}$. Then, $v_{t} v_{n} \in E(G)$ and the size of a minimum $1 \mathrm{PC}$ of $G\left[S-\left\{v_{t}\right\}\right]$ is $\lambda_{\mathcal{T}}(G)-1$, a contradiction.

Suppose that $v_{n}$ is an endpoint of a path $P^{\prime}=\left(v_{n}, v_{a^{\prime}}, \ldots, v_{b^{\prime}}\right) \in \mathcal{P}_{\mathcal{T}}^{\prime}(G)$, such that $v_{a^{\prime}} \notin \mathcal{T}$. Then, removing vertex $v_{n}$ from $\mathcal{P}_{\mathcal{T}}^{\prime}(G)$ results to a $1 \mathrm{PC}$ of $G[S]$ of size $\lambda_{\mathcal{T}}(G[S])$, and, thus, minimum, such that there exists an index $i$ for which $\varepsilon_{i}^{\prime(n-1)}=$ $1, i \geq d$. Then, $d=d^{\prime}$, which is equal to the index of the terminal vertex, and $P^{\prime}$ is the terminal path such that its left endpoint in $\mathcal{P}_{\mathcal{T}}^{\prime}(G[S])$, that is, vertex $v_{a^{\prime}}$, has index greater than the index of the left endpoint of the terminal path in $\mathcal{P}_{\mathcal{T}}(G[S])$. Note that, $v_{a^{\prime}}$ cannot be an endpoint in $\mathcal{P}_{\mathcal{T}}^{\prime}(G[S])$ since $\varepsilon_{i}^{\prime(n-1)} \leq \varepsilon_{i}^{(n-1)}, d \leq i \leq n-1$, and, thus, a $1 \mathrm{PC}$ of $G[S]$ having $v_{a^{\prime}}$ as an endpoint cannot be minimum. However, removing $v_{n}$ from $\mathcal{P}_{\mathcal{T}}^{\prime}(G)$ results to a $1 \mathrm{PC}$ of $G[S]$ of size $\lambda_{\mathcal{T}}(G[S])$ having $v_{a^{\prime}}$ as an endpoint, a contradiction.

Suppose now that $v_{n}$ is not an endpoint in $\mathcal{P}_{\mathcal{T}}^{\prime}(G)$; let $P^{\prime}=\left(\ldots, v_{a^{\prime}}, v_{n}, v_{b^{\prime}}, \ldots\right)$. If $v_{a^{\prime}} v_{b^{\prime}} \in E(G)$ then if we remove $v_{n}$ from $\mathcal{P}_{\mathcal{T}}^{\prime}(G)$ and $\mathcal{P}_{\mathcal{T}}^{\prime}(G)$, we obtain $\varepsilon_{k-1}^{(n-1)}=$ $\varepsilon_{k-1}^{(n)}=\varepsilon_{k-1}^{\prime(n)}-1$ and $\varepsilon_{k-1}^{\prime(n-1)}=\varepsilon_{k-1}^{\prime(n)}$, a contradiction. Consequently, $v_{a^{\prime}} v_{b^{\prime}} \notin E(G)$. Suppose that the value of $d$-connectivity of $\mathcal{P}_{\mathcal{T}}(G[S])$ is $c$; then the value of $d$ connectivity of $\mathcal{P}_{\mathcal{T}}(G)$ is $c+2$. However, the corresponding value of $\mathcal{P}_{\mathcal{T}}^{\prime}(G)$ is not increased by vertices $v_{a^{\prime}}, v_{n}$ and $v_{b^{\prime}}$, since $v_{a^{\prime}}$ and $v_{b^{\prime}}$ are internal vertices not successive into a path in a 1PC of $G[S]$ and there exist two vertices connected to $v_{a^{\prime}}$ and $v_{b^{\prime}}$ in $\mathcal{P}_{\mathcal{T}}(G[S])$, say, $v_{f}$ and $v_{g}$, respectively, for which $d\left(v_{f}\right)$ and $d\left(v_{g}\right)$ are reduced, and, thus, they reduce the $d$-connectivity by two. In order to obtain $c+2$ for $\mathcal{P}_{\mathcal{T}}^{\prime}(G)$ the vertices of $V(G)-\left\{v_{a^{\prime}}, v_{b^{\prime}}, v_{n}\right\}$ must increase the $d$-connectivity by two. However, the size of $\mathcal{P}_{\mathcal{T}}^{\prime}(G)$ is also $\lambda_{\mathcal{T}}(G)$ and vertices $v_{a^{\prime}}, v_{b^{\prime}}$ and $v_{n}$ are also internal in $\mathcal{P}_{\mathcal{T}}(G)$. Thus, increasing the $d$-connectivity by two is not possible and we have a contradiction. Note that $v_{f} v_{g} \notin E(G)$; otherwise we would have also $v_{n} v_{f} \in E(G)$ and $v_{n} v_{g} \in E(G)$ and there would exist a $1 \mathrm{PC}$ having the same endpoints as $\mathcal{P}_{\mathcal{T}}^{\prime}(G)$ and containing a path $P=\left(\ldots, v_{f}, v_{n}, v_{g}, \ldots\right)$ with $v_{f} v_{g} \in E(G)$, a contradiction.

Case B.2. Suppose that $\varepsilon_{i}^{(n-1)}=\varepsilon_{i}^{(n-1)}+1, d \leq i<d^{\prime}$ and $\varepsilon_{i}^{\prime(n-1)}=\varepsilon_{i}^{(n-1)}$, $d^{\prime} \leq i \leq n-1$ and there exists a vertex $v_{q}$ such that $\varepsilon_{q}^{(n-1)}>\varepsilon_{q}^{\prime(n-1)}, 1 \leq q<d$ 
and there exists no vertex $v_{q^{\prime}}, 1 \leq q^{\prime}<d$, such that $\varepsilon_{q^{\prime}}^{\prime(n-1)}>\varepsilon_{q^{\prime}}^{(n-1)}$. Using similar arguments as in Case A.1 where $v_{n}$ is not an endpoint in $\mathcal{P}_{\mathcal{T}}(G[S])$, we show that the algorithm computes a minimum $1 \mathrm{PC} \mathcal{P}_{\mathcal{T}}(G)$ of the graph $G$ having $\varepsilon_{i}^{(n)}$ endpoints $v_{\kappa}$ belonging to different paths with index $\kappa \in(i, n], 1 \leq i \leq n$, such that there is no other minimum $1 \mathrm{PC} \mathcal{P}_{\mathcal{T}}^{\prime}(G)$ having $\varepsilon_{i}^{\prime(n)}$ endpoints $v_{\mathcal{K}^{\prime}}$ belonging to different paths with index $\kappa^{\prime} \in(i, n]$ such that $\varepsilon_{i}^{\prime(n)}>\varepsilon_{i}^{(n)}, 1 \leq i<\varrho$. Furthermore, if $\varepsilon_{i}^{\prime(n)}=\varepsilon_{i}^{(n)}+1$, $\varrho \leq i<\varrho^{\prime}$ and $\varepsilon_{i}^{\prime(n)}=\varepsilon_{i}^{(n)}, \varrho^{\prime} \leq i \leq n$ then there exists a vertex $v_{z}$ such that $\varepsilon_{z}^{(n)}>$ $\varepsilon_{z}^{\prime(n)}, 1 \leq z<\varrho$ and there exists no vertex $v_{z^{\prime}}, 1 \leq z^{\prime}<\varrho$, such that $\varepsilon_{z^{\prime}}^{(n)}>\varepsilon_{z^{\prime}}^{(n)}$.

Lemma 4.5 Let $G$ be an interval graph and suppose that Algorithm 1PC_Interval computes a minimum $1 \mathrm{PC} \mathcal{P}_{\mathcal{T}}(G[S])$ of the graph $G[S], S=\left\{v_{1}, v_{2}, \ldots, v_{n-1}\right\}$, on at most $n-1$ vertices satisfying Property 1 PC-on- $G[S]$. Let $\lambda_{\mathcal{T}}(G[S])$ be the size of $\mathcal{P}_{\mathcal{T}}(G[S])$ and let $v_{n}$ be a non-terminal vertex. If during the process of vertex $v_{n}$ the algorithm creates a new path having vertex $v_{n}$ as an endpoint then Algorithm 1PC_Interval computes a minimum $1 \mathrm{PC} \mathcal{P}_{\mathcal{T}}(G)$ of the graph $G$ satisfying Property 1 PC-on- $G$.

Proof Algorithm 1PC_InTERVAL computes a minimum 1PC $\mathcal{P}_{\mathcal{T}}(G[S])$ of the interval graph $G[S], S=\left\{v_{1}, v_{2}, \ldots, v_{n-1}\right\}$, on at most $n-1$ vertices having $\varepsilon_{i}^{(n-1)}$ endpoints $v_{\kappa}$ belonging to different paths with index $\kappa \in(i, n-1], 1 \leq i \leq n-1$, such that there is no other minimum $1 \mathrm{PC} \mathcal{P}_{\mathcal{T}}^{\prime}(G[S])$ having $\varepsilon_{i}^{\prime(n-1)}$ endpoints $v_{\kappa^{\prime}}$ belonging to different paths with index $\kappa^{\prime} \in(i, n-1]$ such that $\varepsilon_{i}^{\prime(n-1)}>\varepsilon_{i}^{(n-1)}$, $1 \leq i<d$, where $d$ is the index of the rightmost endpoint of a path in $\mathcal{P}_{\mathcal{T}}(G[S])$, and, moreover, exactly one of the following holds:

(i) $\varepsilon_{i}^{\prime(n-1)} \leq \varepsilon_{i}^{(n-1)}, d \leq i \leq n-1$;

(ii) $\varepsilon_{i}^{\prime(n-1)}=\varepsilon_{i}^{(n-1)}+1, d \leq i<d^{\prime}$ and $\varepsilon_{i}^{\prime(n-1)}=\varepsilon_{i}^{(n-1)}, d^{\prime} \leq i \leq n-1$, where $d^{\prime}$ is the index of the rightmost endpoint of a path in $\mathcal{P}_{\mathcal{T}}^{\prime}(G[S])$, and there exists a vertex $v_{q}$ such that $\varepsilon_{q}^{(n-1)}>\varepsilon_{q}^{\prime(n-1)}$, and there exists no vertex $v_{q^{\prime}}$ such that $\varepsilon_{q^{\prime}}^{\prime(n-1)}>\varepsilon_{q^{\prime}}^{(n-1)}, 1 \leq q, q^{\prime}<d$.

When the algorithm processes vertex $v_{n}$, it creates a new path having vertex $v_{n}$ as an endpoint, that is, $\lambda_{\mathcal{T}}(G)=\lambda_{\mathcal{T}}(G[S])+1$. This implies that $\forall i \geq d$ we have $\varepsilon_{i}^{(n-1)} \leq 1$. Suppose that there exists a $1 \mathrm{PC} \mathcal{P}_{\mathcal{T}}^{\prime}(G)$ of size $\lambda_{\mathcal{T}}(G[S])-1$, that is, vertex $v_{n}$ is an internal vertex of a path $P$ in $\mathcal{P}_{\mathcal{T}}^{\prime}(G)$. Then, removing vertex $v_{n}$ from $\mathcal{P}_{\mathcal{T}}^{\prime}(G)$ results to a $1 \mathrm{PC}$ of $G[S]$ of size $\lambda_{\mathcal{T}}(G[S])$, and, thus, minimum, such that there exists an index $i$ for which $\varepsilon_{i}^{\prime(n-1)}=2$, a contradiction.

Suppose now that there exists a $1 \mathrm{PC} \mathcal{P}_{\mathcal{T}}^{\prime}(G)$ of size $\lambda_{\mathcal{T}}(G[S])$. Let $v_{n}$ be an endpoint of a path $P$ in $\mathcal{P}_{\mathcal{T}}^{\prime}(G)$. We distinguish the following cases:

(i) $P=\left(v_{n}, v_{r}, \ldots, v_{s}\right)$. Removing vertex $v_{n}$ from $\mathcal{P}_{\mathcal{T}}^{\prime}(G)$ results to a $1 \mathrm{PC}$ of $G[S]$ of size $\lambda_{\mathcal{T}}(G[S])$, and, thus, minimum, such that there exists an index $i$ for which $\varepsilon_{i}^{\prime(n-1)}=1, i \geq d$. Then, $d=d^{\prime}$, which is equal to the index of the terminal vertex, and $P$ is the terminal path such that its left endpoint in $\mathcal{P}_{\mathcal{T}}^{\prime}(G[S])$, that is, vertex $v_{r}$, has index greater than the index of the left endpoint of the terminal path in 
$\mathcal{P}_{\mathcal{T}}(G[S])$. Note that, $v_{r}$ cannot be an endpoint in $\mathcal{P}_{\mathcal{T}}^{\prime}(G[S])$ since $\varepsilon_{i}^{\prime(n-1)} \leq \varepsilon_{i}^{(n-1)}$, $d \leq i \leq n-1$, and, thus, a $1 \mathrm{PC}$ of $G[S]$ having $v_{r}$ as an endpoint cannot be minimum. However, removing $v_{n}$ from $\mathcal{P}_{\mathcal{T}}^{\prime}(G)$ results to a $1 \mathrm{PC}$ of $G[S]$ of size $\lambda_{\mathcal{T}}(G[S])$ having $v_{r}$ as an endpoint, a contradiction.

(ii) $P=\left(v_{t}, v_{n}\right)$, where $v_{t}$ is the terminal vertex. Removing $v_{n}$ and $v_{t}$ from $P$ results to a 1PC of $G\left[S-\left\{v_{t}\right\}\right]$ of size $\lambda_{\mathcal{T}}(G[S])-1$, a contradiction. Indeed, since the algorithm does not use $v_{n}$ to bridge two paths, removing $v_{t}$ from $G[S]$ results to $\lambda_{\mathcal{T}}(G[S])$ paths.

Now let $v_{n}$ be an internal vertex of a path $P=\left(v_{r}, \ldots, v_{i}, v_{n}, v_{j}, \ldots, v_{s}\right)$ in $\mathcal{P}_{\mathcal{T}}^{\prime}(G)$. Suppose that $N\left(v_{n}\right)>0$ (the case where $N\left(v_{n}\right)=0$ is trivial) and $v_{t} \notin$ $N\left(v_{n}\right)$, where $v_{t}$ is the terminal vertex. Since the algorithm constructs $\lambda_{\mathcal{T}}(G[S])+1$ paths, at least $\left|N\left(v_{n}\right)\right|-1$ neighbors of $v_{n}$ have bridged paths reducing the size of the 1PC and at most one of them was inserted; otherwise there would exist at least two successive neighbors into a path or at least one of them would be an endpoint. Suppose that $v_{i}$ and $v_{j}$ have both bridged paths. Then, applying the algorithm to $G-\left\{v_{i}, v_{j}, v_{n}\right\}$ would result to a minimum 1PC of $G-\left\{v_{i}, v_{j}, v_{n}\right\}$ of size $\lambda_{\mathcal{T}}(G[S])+2$. However, if we remove vertices $v_{i}, v_{j}$ and $v_{n}$ from $\mathcal{P}_{\mathcal{T}}^{\prime}(G)$ we obtain a $1 \mathrm{PC}$ of $G-\left\{v_{i}, v_{j}, v_{n}\right\}$ of size $\lambda_{\mathcal{T}}(G[S])+1$, a contradiction. Suppose now that $v_{i}$ was inserted and $v_{j}$ has bridged paths. We distinguish the following cases:

(i) $j<i$. Clearly, applying the algorithm to $G^{\prime}=G-\left\{v_{i}, v_{n}\right\}$ results to a minimum 1PC of $G^{\prime}$ of size $\lambda_{\mathcal{T}}(G[S])$. Furthermore, applying the algorithm to $G^{\prime}-\left\{v_{j}\right\}$ results to a minimum $1 \mathrm{PC} \mathcal{P}^{\prime \prime}{ }_{1}(G)$ of $G^{\prime}-\left\{v_{j}\right\}$ of size $\lambda_{\mathcal{T}}(G[S])+1$ such that no non-terminal neighbor of $v_{i}$ is an endpoint and if $v_{i}$ sees the terminal vertex $v_{t}$, it is not a trivial path in $\mathcal{P}^{\prime \prime}{ }_{1}(G)$. Indeed, any neighbor $v_{a}$ of $v_{i}$ such that $i<a<n$ cannot be an endpoint in $\mathcal{P}^{\prime \prime}{ }_{1}(G)$ since every vertex $v_{a}$ such that $i<a<n$ is also a neighbor of $v_{n}$. Note that, $t<j$. Furthermore, since $v_{i}$ is inserted, when vertex $v_{j+1}$ was processed, no neighbor of $v_{i}$ was an endpoint and if $v_{i} v_{t} \in E(G)$ vertex $v_{t}$ does not belong to a trivial path. Indeed, let $v_{k} \in N\left(v_{i}\right)$ be an endpoint when the algorithm processes vertex $v_{j+1}$ or $v_{t}$ belongs to a trivial path. This implies that, when we apply the algorithm to $G[S]$, we have one neighbor of $v_{n}$, say, $v_{\ell}$, bridging through vertex $v_{k}$ or vertex $v_{t}$; then $v_{i}$ would be inserted through the edge $v_{k} v_{\ell}$ or $v_{t} v_{\ell}$, which is a contradiction since this results to two neighbors of $v_{n}$ being successive. Additionally, no neighbor of $v_{i}$ becomes an endpoint and vertex $v_{t}$ does not belong to a trivial path until vertex $v_{n-1}$ is processed, since all vertices with index greater than $j+1$ are neighbors of $v_{n}$, and, thus, they are used to bridge paths reducing the size of the 1PC. Note that, according to the algorithm, vertex $v_{t}$ cannot belong to a trivial path until vertex $v_{n-1}$ is processed, since no bridge operation results to $v_{t}$ belonging to a trivial path. Consequently, applying the algorithm to $G^{\prime}-\left\{v_{j}\right\}$ results to a minimum 1PC $\mathcal{P}^{\prime \prime}{ }_{1}\left(G^{\prime}-\left\{v_{j}\right\}\right)$ of size $\lambda_{\mathcal{T}}(G[S])+1$ such that no non-terminal neighbor of $v_{i}$ is an endpoint and if $v_{i}$ sees the terminal vertex $v_{t}$, it is not a trivial path in $\mathcal{P}^{\prime \prime}{ }_{1}\left(G^{\prime}-\left\{v_{j}\right\}\right)$.

(ii) $i<j$. Similarly to case (i), applying the algorithm to $G^{\prime}-\left\{v_{j}\right\}$ results to a minimum $1 \mathrm{PC} \mathcal{P}^{\prime \prime}{ }_{1}\left(G^{\prime}-\left\{v_{j}\right\}\right)$ of size $\lambda_{\mathcal{T}}(G[S])+1$ such that no non-terminal neighbor of $v_{i}$ is an endpoint and if $v_{i}$ sees the terminal vertex $v_{t}$, it is not a trivial path in $\mathcal{P}^{\prime \prime}{ }_{1}\left(G^{\prime}-\left\{v_{j}\right\}\right)$. Indeed, when vertex $v_{i+1}$ is processed, no neighbor of $v_{i}$ is an endpoint and if $v_{i}$ sees the terminal vertex $v_{t}$, it is not a trivial path. Furthermore, since no neighbor of $v_{n}$ can be an endpoint, no vertex with index greater than $i+1$ is 
an endpoint. Additionally, no neighbor of $v_{i}$ becomes an endpoint and if $v_{t} v_{i} \in E(G)$, vertex $v_{t}$ does not belong to a trivial path until vertex $v_{n-1}$ is processed, since all vertices with index greater than $i+1$ are neighbors of $v_{n}$, and, thus, they are used to bridge paths reducing the size of the 1PC. Note that, according to the algorithm, vertex $v_{t}$ cannot belong to a trivial path until vertex $v_{n-1}$ is processed. Consequently, applying the algorithm to $G^{\prime}-\left\{v_{j}\right\}$ results to a minimum $1 \mathrm{PC} \mathcal{P}^{\prime \prime}{ }_{1}\left(G^{\prime}-\left\{v_{j}\right\}\right)$ of size $\lambda_{\mathcal{T}}(G[S])+1$ such that no non-terminal neighbor of $v_{i}$ is an endpoint and if $v_{i}$ sees the terminal vertex $v_{t}$, it is not a trivial path in $\mathcal{P}^{\prime \prime}{ }_{1}\left(G^{\prime}-\left\{v_{j}\right\}\right)$.

Since $v_{n}$ is an internal vertex of a path $P=\left(v_{r}, \ldots, v_{i}, v_{n}, v_{j}, \ldots, v_{s}\right)$ in $\mathcal{P}_{\mathcal{T}}^{\prime}(G)$ which has size $\lambda_{\mathcal{T}}(G[S])$, if we remove vertices $v_{i}, v_{j}$ and $v_{n}$ from $P$ we obtain a 1PC of $G^{\prime}-\left\{v_{j}\right\}$ of size $\lambda_{\mathcal{T}}(G[S])+1$ such that a non-terminal neighbor of $v_{i}$ is an endpoint, a contradiction; the same holds when $P=\left(v_{r}, \ldots, v_{i}, v_{n}, v_{j}, v_{t}\right)$. If $P=$ $\left(v_{t}, v_{i}, v_{n}, v_{j}, \ldots, v_{s}\right)$ then $v_{t}$ belongs to a trivial path in $\mathcal{P}^{\prime \prime}{ }_{1}(G)$, a contradiction. If $P=\left(v_{i}, v_{n}, v_{j}, \ldots, v_{s}\right)$ or $P=\left(v_{r}, \ldots, v_{i}, v_{n}, v_{j}\right)$, then removing vertices $v_{i}, v_{j}$ and $v_{n}$ from $P$ results to a $1 \mathrm{PC}$ of $G^{\prime}-\left\{v_{j}\right\}$ of size $\lambda_{\mathcal{T}}(G[S])$, a contradiction.

Now let $v_{t} \in N\left(v_{n}\right)$, where $v_{t}$ is the terminal vertex. The case where $v_{n}$ is an internal vertex of a path $P=\left(v_{r}, \ldots, v_{i}, v_{n}, v_{j}, \ldots, v_{s}\right)$ in $\mathcal{P}_{\mathcal{T}}^{\prime}(G)$ which has size $\lambda_{\mathcal{T}}(G[S])$ leads to a contradiction similarly to the case where $v_{t} \notin N\left(v_{n}\right)$. Suppose that $v_{n}$ is an internal vertex of a path $P=\left(v_{t}, v_{n}, v_{j}, \ldots, v_{s}\right)$ in $\mathcal{P}_{\mathcal{T}}^{\prime}(G)$ which has size $\lambda_{\mathcal{T}}(G[S])$. According to the algorithm, no neighbor of $v_{n}$ is inserted until vertex $v_{t}$ is processed. Also, it is easy to see that, no neighbor of $v_{n}$ with index greater than $t$ is inserted, either. Indeed, let $v_{a}, t<a<n$, be a neighbor of $v_{n}$ which is inserted into the terminal path. Since the algorithm results to $\lambda_{\mathcal{T}}(G[S])+1$ paths, $v_{a}$ is inserted through an edge $v_{k} v_{\ell}$ such that $v_{k}, v_{\ell} \notin N\left(v_{n}\right)$ and $v_{t}$ is connected to a vertex $v_{q}$ such that $v_{q} \notin N\left(v_{n}\right)$. This implies that the ordering of the vertices $v_{t}, v_{k}, v_{\ell}$ and $v_{q}$ of the terminal path is as follows: $v_{q}<v_{k}<v_{\ell}<v_{t}$ or $v_{q}<v_{\ell}<v_{k}<v_{t}$. Without loss of generality suppose that $v_{q}<v_{k}<v_{\ell}<v_{t}$. Let $v_{b}$ be the other endpoint of the terminal path. Clearly $v_{b}<v_{k}$. Also, without loss of generality, suppose that $v_{b}<v_{q}$. Consequently, when vertex $v_{t}$ is processed, the algorithm has constructed a path having two successive vertices, $v_{k}$ and $v_{\ell}$, which have indexes greater than those of the endpoints of the path, that is, $v_{b}$ and $v_{q}$. This is a contradiction, since it implies that there exists at least one vertex with index greater than $q$ which sees $v_{q}$; in this case the algorithm could not result to a path having $v_{q}$ as an endpoint. Consequently, we have shown that if we apply the algorithm to $G[S]$, no neighbor of $v_{n}$ is inserted into the terminal path. Furthermore, since there are no neighbors of $v_{n}$ successive into a path, all neighbors of $v_{n}$ bridge paths reducing the size of the 1PC. This implies that, if we apply the algorithm to $G\left[S-\left\{v_{t}\right\}\right]$, we obtain a minimum 1PC of $G\left[S-\left\{v_{t}\right\}\right]$ of size $\lambda_{\mathcal{T}}(G[S])$. Furthermore, if we apply the algorithm to $G\left[S-\left\{v_{t}, v_{j}\right\}\right]$, we obtain a minimum $1 \mathrm{PC}$ of $G\left[S-\left\{v_{t}, v_{j}\right\}\right]$ of size $\lambda_{\mathcal{T}}(G[S])+1$. However, removing $v_{t}, v_{n}$ and $v_{j}$ from $P=\left(v_{t}, v_{n}, v_{j}, \ldots, v_{s}\right)$ which is a path in $\mathcal{P}_{\mathcal{T}}^{\prime}(G)$, we obtain a $1 \mathrm{PC}$ of $G\left[S-\left\{v_{t}, v_{j}\right\}\right]$ of size at most $\lambda_{\mathcal{T}}(G[S])$, a contradiction; thus, $v_{n}$ cannot be an internal vertex of a path $P=\left(v_{t}, v_{n}, v_{j}, \ldots, v_{s}\right)$ in $\mathcal{P}_{\mathcal{T}}^{\prime}(G)$ which has size $\lambda_{\mathcal{T}}(G[S])$.

We have shown that there does not exist a $1 \mathrm{PC} \mathcal{P}_{\mathcal{T}}^{\prime}(G)$ of size $\lambda_{\mathcal{T}}(G[S])$, and, thus, $\mathcal{P}_{\mathcal{T}}(G)$ is a minimum $1 \mathrm{PC}$ of $G$, that is, $\lambda_{\mathcal{T}}^{\prime}(G)=\lambda_{\mathcal{T}}(G)=\lambda_{\mathcal{T}}(G[S])+1$.

Using similar arguments as in Case A.1 where $v_{n}$ is an endpoint in $\mathcal{P}_{\mathcal{T}}(G[S])$, we show that the algorithm computes a minimum $1 \mathrm{PC} \mathcal{P}_{\mathcal{T}}(G)$ of every interval 
graph $G$ with $n$ vertices having $\varepsilon_{i}^{(n)}$ endpoints $v_{\kappa}$ belonging to different paths with index $\kappa \in(i, n], 1 \leq i \leq n$, such that there is no other minimum $1 \mathrm{PC} \mathcal{P}_{\mathcal{T}}^{\prime}(G)$ having $\varepsilon_{i}^{\prime(n)}$ endpoints $v_{\mathcal{K}^{\prime}}$ belonging to different paths with index $\kappa^{\prime} \in(i, n]$ such that $\varepsilon_{i}^{\prime(n)}>\varepsilon_{i}^{(n)}, 1 \leq i \leq n$.

Lemma 4.6 Let $G$ be an interval graph and suppose that Algorithm 1PC_INTERVAL computes a minimum $1 \mathrm{PC} \mathcal{P}_{\mathcal{T}}(G[S])$ of the graph $G[S], S=\left\{v_{1}, v_{2}, \ldots, v_{n-1}\right\}$, on at most $n-1$ vertices satisfying Property $1 \mathrm{PC}-\mathrm{on}-G[S]$. Let $\lambda_{\mathcal{T}}(G[S])$ be the size of $\mathcal{P}_{\mathcal{T}}(G[S])$ and let $v_{n}$ be the terminal vertex; then Algorithm 1PC_INTERVAL computes a minimum $1 \mathrm{PC} \mathcal{P}_{\mathcal{T}}(G)$ of the graph $G$ satisfying Property $1 \mathrm{PC}-\mathrm{on}-G$.

Proof Clearly, the size $\lambda_{\mathcal{T}}^{\prime}(G)$ of a minimum $1 \mathrm{PC}$ of $G$ is equal to $\lambda_{\mathcal{T}}(G[S])$ or $\lambda_{\mathcal{T}}(G[S])+1$. We distinguish the following cases:

Case 1 When the algorithm processes vertex $v_{n}$, it connects $v_{n}$ to a path, that is, $\lambda_{\mathcal{T}}(G)=\lambda_{\mathcal{T}}(G[S])$. Since $v_{n}$ is the terminal vertex, the $1 \mathrm{PC} \mathcal{P}_{\mathcal{T}}(G)$ is a minimum $1 \mathrm{PC}$ of $G$, that is, $\lambda_{\mathcal{T}}^{\prime}(G)=\lambda_{\mathcal{T}}(G)=\lambda_{\mathcal{T}}(G[S])$.

We show that the algorithm computes a minimum 1 PC $\mathcal{P}_{\mathcal{T}}(G)$ of the graph $G$ having $\varepsilon_{i}^{(n)}$ endpoints $v_{\kappa}$ belonging to different paths with index $\kappa \in(i, n], 1 \leq i \leq n$, such that there is no other minimum $1 \mathrm{PC} \mathcal{P}_{\mathcal{T}}^{\prime}(G)$ having $\varepsilon_{i}^{\prime(n)}$ endpoints $v_{\mathcal{K}^{\prime}}$ belonging to different paths with index $\kappa^{\prime} \in(i, n]$ such that $\varepsilon_{i}^{\prime(n)}>\varepsilon_{i}^{(n)}, 1 \leq i \leq n$.

Suppose that $v_{n} \in P=\left(v_{n}, v_{a}, \ldots\right) \in \mathcal{P}_{\mathcal{T}}(G)$. Then, there cannot exist a minimum $1 \mathrm{PC} \mathcal{P}_{\mathcal{T}}^{\prime}(G)$ such that $1=\varepsilon_{\varrho-1}^{(n)}<\varepsilon_{\varrho-1}^{\prime(n)}$. Assume that there exists a minimum 1PC $\mathcal{P}_{\mathcal{T}}^{\prime}(G)$ having an index, say, $k-1$, for which we have $\varepsilon_{k-1}^{\prime(n)}$ endpoints $v_{\kappa^{\prime}}$ belonging to different paths with index $\kappa^{\prime} \in(k-1, n]$, where $\varepsilon_{k-1}^{\prime(n)}>\varepsilon_{k-1}^{(n)}$. Similarly, to Case 1 of Lemma 4.1, there exists a vertex $v_{j}, 1 \leq j<k-1$, such that $\varepsilon_{j}^{(n)}=\varepsilon_{j}^{\prime(n)}$.

Suppose that $v_{n} \in P^{\prime}=\left(v_{n}, v_{a^{\prime}}, \ldots\right) \in \mathcal{P}_{\mathcal{T}}^{\prime}(G)$. If $v_{a^{\prime}}<v_{j+1}$, then $v_{j+1} v_{n} \in$ $E(G)$, and, thus, $v_{a}<v_{j+1}$. Then, if we remove $v_{n}$ from $\mathcal{P}_{\mathcal{T}}(G)$ and $\mathcal{P}_{\mathcal{T}}^{\prime}(G)$, we obtain $\varepsilon_{j+1}^{(n-1)}=\varepsilon_{j+1}^{(n)}-1=\varepsilon_{j+1}^{\prime(n)}-2$ and $\varepsilon_{j+1}^{\prime(n-1)}=\varepsilon_{j+1}^{\prime(n)}-1$; thus, $\varepsilon_{j+1}^{(n-1)}=\varepsilon_{j+1}^{\prime(n-1)}-1$, a contradiction. On the other hand, if $v_{a^{\prime}}>v_{j+1}$, then, if we remove $v_{n}$ from $\mathcal{P}_{\mathcal{T}}(G)$ and $\mathcal{P}_{\mathcal{T}}^{\prime}(G)$, we obtain $\varepsilon_{j+1}^{(n-1)}=\varepsilon_{j+1}^{(n)}-1=\varepsilon_{j+1}^{\prime(n)}-2$ or $\varepsilon_{j+1}^{(n-1)}=\varepsilon_{j+1}^{(n)}=\varepsilon_{j+1}^{\prime(n)}-1$. Also, $\varepsilon_{j+1}^{\prime(n-1)}=\varepsilon_{j+1}^{\prime(n)}$; thus, $\varepsilon_{j+1}^{(n-1)}=\varepsilon_{j+1}^{\prime(n-1)}-1$, a contradiction.

Case 2 When the algorithm processes vertex $v_{n}$, it constructs a new trivial path, that is, $\lambda_{\mathcal{T}}(G)=\lambda_{\mathcal{T}}(G[S])+1$. Suppose that there exists a $1 \mathrm{PC} \mathcal{P}_{\mathcal{T}}^{\prime}(G)$ of size $\lambda_{\mathcal{T}}(G[S])$. Clearly, vertex $v_{n}$ cannot belong to a trivial path in $\mathcal{P}_{\mathcal{T}}^{\prime}(G)$, since removing it results to a $1 \mathrm{PC}$ of $G[S]$ of size $\lambda_{\mathcal{T}}(G[S])-1$, a contradiction. Thus, let $P=\left(v_{n}, v_{r}, \ldots\right) \in \mathcal{P}_{\mathcal{T}}^{\prime}(G)$ be the path containing $v_{n}$. Removing vertex $v_{n}$ from $\mathcal{P}_{\mathcal{T}}^{\prime}(G)$ results to a $1 \mathrm{PC}$ of $G[S]$ of size $\lambda_{\mathcal{T}}(G[S])$, and, thus, minimum, having a neighbor of $v_{n}$, that is, vertex $v_{r}$, as an endpoint of a path. Since $G[S]$ does not contain the terminal vertex this is a contradiction. Consequently, the $1 \mathrm{PC} \mathcal{P}_{\mathcal{T}}(G)$ is a minimum $1 \mathrm{PC}$ of $G$, that is, $\lambda_{\mathcal{T}}^{\prime}(G)=\lambda_{\mathcal{T}}(G)=\lambda_{\mathcal{T}}(G[S])+1$.

We show that the algorithm computes a minimum 1 PC $\mathcal{P}_{\mathcal{T}}(G)$ of the graph $G$ having $\varepsilon_{i}^{(n)}$ endpoints $v_{\kappa}$ belonging to different paths with index $\kappa \in(i, n], 1 \leq i \leq n$, 
such that there is no other minimum $1 \mathrm{PC} \mathcal{P}_{\mathcal{T}}^{\prime}(G)$ having $\varepsilon_{i}^{\prime(n)}$ endpoints $v_{\kappa^{\prime}}$ belonging to different paths with index $\kappa^{\prime} \in(i, n]$ such that $\varepsilon_{i}^{\prime(n)}>\varepsilon_{i}^{(n)}, 1 \leq i \leq n$.

Since $P=\left(v_{n}\right)$ there cannot exist a minimum $1 \mathrm{PC} \mathcal{P}_{\mathcal{T}}^{\prime}(G)$ such that $1=\varepsilon_{\varrho-1}^{(n)}<$ $\varepsilon_{\varrho-1}^{\prime(n)}$. Assume that there exists a minimum $1 \mathrm{PC} \mathcal{P}_{\mathcal{T}}^{\prime}(G)$ having an index, say, $k-1$, for which we have $\varepsilon_{k-1}^{\prime(n)}$ endpoints $v_{\kappa^{\prime}}$ belonging to different paths with index $\kappa^{\prime} \in$ $(k-1, n]$, where $\varepsilon_{k-1}^{\prime(n)}>\varepsilon_{k-1}^{(n)}$. Suppose that $\varepsilon_{k-1}^{(n)}=x$ and $\varepsilon_{k-1}^{\prime(n)}=x+1$. Similarly, to Case 1 of Lemma 4.1, there exists a vertex $v_{j}, 1 \leq j<k-1$, such that $\varepsilon_{j}^{(n)}=\varepsilon_{j}^{(n)}$.

Suppose that $v_{n} \in P^{\prime}=\left(v_{n}, v_{a^{\prime}}, \ldots\right) \in \mathcal{P}_{\mathcal{T}}^{\prime}(G)$; the case where $P^{\prime}=\left(v_{n}\right)$ is trivial. If $v_{a^{\prime}}<v_{j+1}$, then $v_{j+1} v_{n} \in E(G)$, and, thus, vertex $v_{n}$ would be connected, a contradiction. If $v_{a^{\prime}}>v_{j+1}$, then, if we remove $v_{n}$ from $\mathcal{P}_{\mathcal{T}}(G)$ and $\mathcal{P}_{\mathcal{T}}^{\prime}(G)$, we obtain $\varepsilon_{j+1}^{(n-1)}=\varepsilon_{j+1}^{(n)}-1=\varepsilon_{j+1}^{\prime(n)}-2$ and $\varepsilon_{j+1}^{\prime(n-1)}=\varepsilon_{j+1}^{\prime(n)}$, a contradiction.

Consequently, we prove the following theorem.

Theorem 4.1 Let $G$ be an interval graph on $n$ vertices and $m$ edges and let $\mathcal{T}=\{v\}$, where $v \in V(G)$. Algorithm 1PC_Interval computes a minimum $1 \mathrm{PC} \mathcal{P}_{\mathcal{T}}(G)$ of the graph $G$ satisfying Property $1 \mathrm{PC}-\mathrm{on}-G$.

Proof We use induction on $n$. The basis $n=1$ is trivial. Assume now that Algorithm 1 PC_INTERVAL computes a minimum $1 \mathrm{PC} \mathcal{P}_{\mathcal{T}}(G[S])$ of every interval graph $G[S]$, $S=\left\{v_{1}, v_{2}, \ldots, v_{n-1}\right\}$, on at most $n-1$ vertices having $\varepsilon_{i}^{(n-1)}$ endpoints $v_{\kappa}$ belonging to different paths with index $\kappa \in(i, n-1], 1 \leq i \leq n-1$, such that there is no other minimum $1 \mathrm{PC} \mathcal{P}_{\mathcal{T}}^{\prime}(G[S])$ having $\varepsilon_{i}^{\prime(n-1)}$ endpoints $v_{\kappa^{\prime}}$ belonging to different paths with index $\kappa^{\prime} \in(i, n-1]$ such that $\varepsilon_{i}^{\prime(n-1)}>\varepsilon_{i}^{(n-1)}, 1 \leq i<d$, where $d$ is the index of the rightmost endpoint of a path in $\mathcal{P}_{\mathcal{T}}(G[S])$, and, moreover, exactly one of the following holds:

(i) $\varepsilon_{i}^{\prime(n-1)} \leq \varepsilon_{i}^{(n-1)}, d \leq i \leq n-1$;

(ii) $\varepsilon_{i}^{\prime(n-1)}=\varepsilon_{i}^{(n-1)}+1, d \leq i<d^{\prime}$ and $\varepsilon_{i}^{\prime(n-1)}=\varepsilon_{i}^{(n-1)}, d^{\prime} \leq i \leq n-1$, where $d^{\prime}$ is the index of the rightmost endpoint of a path in $\mathcal{P}_{\mathcal{T}}^{\prime}(G[S])$, and there exists a vertex $v_{q}$ such that $\varepsilon_{q}^{(n-1)}>\varepsilon_{q}^{\prime(n-1)}$, and there exists no vertex $v_{q^{\prime}}$ such that $\varepsilon_{q^{\prime}}^{(n-1)}>\varepsilon_{q^{\prime}}^{(n-1)}, 1 \leq q, q^{\prime}<d$.

Let $\lambda_{\mathcal{T}}(G[S])$ be the size of $\mathcal{P}_{\mathcal{T}}(G[S])$. Using Lemmas 4.1-4.6, we show that the algorithm computes a minimum $1 \mathrm{PC} \mathcal{P}_{\mathcal{T}}(G)$ of the graph $G$ having $\varepsilon_{i}^{(n)}$ endpoints $v_{\kappa}$ belonging to different paths with index $\kappa \in(i, n], 1 \leq i \leq n$, such that there is no other minimum $1 \mathrm{PC} \mathcal{P}_{\mathcal{T}}^{\prime}(G)$ having $\varepsilon_{i}^{\prime(n)}$ endpoints $v_{\mathcal{K}^{\prime}}$ belonging to different paths with index $\kappa^{\prime} \in(i, n]$ such that $\varepsilon_{i}^{\prime(n)}>\varepsilon_{i}^{(n)}, 1 \leq i<\varrho$, where $\varrho$ is the index of the rightmost endpoint of a path in $\mathcal{P}_{\mathcal{T}}(G)$, and, moreover, exactly one of the following holds: 
(i) $\varepsilon_{i}^{\prime(n)} \leq \varepsilon_{i}^{(n)}, \varrho \leq i \leq n$;

(ii) $\varepsilon_{i}^{\prime(n)}=\varepsilon_{i}^{(n)}+1, \varrho \leq i<\varrho^{\prime}$ and $\varepsilon_{i}^{\prime(n)}=\varepsilon_{i}^{(n)}, \varrho^{\prime} \leq i \leq n$, where $\varrho^{\prime}$ is the index of the rightmost endpoint of a path in $\mathcal{P}_{\mathcal{T}}^{\prime}(G)$, and there exists a vertex $v_{z}$ such that $\varepsilon_{z}^{(n)}>\varepsilon_{z}^{\prime(n)}$, and there exists no vertex $v_{z^{\prime}}$ such that $\varepsilon_{z^{\prime}}^{\prime(n)}>\varepsilon_{z^{\prime}}^{(n)}, 1 \leq z, z^{\prime}<\varrho$.

Thus the theorem follows.

Let us now compute the time and space complexity of Algorithm 1PC_INTERVAL. As mentioned in the description of the algorithm, it constructs the ordering $\pi=$ $\left(v_{1}, v_{2}, \ldots, v_{n}\right)$ of the vertices of the input graph $G$ and, using a greedy approach on $\pi$, computes a minimum $1 \mathrm{PC} \mathcal{P}(G)$ of the interval graph $G$. In particular, it visits the vertices in the ordering $\pi=\left(v_{1}, \ldots, v_{i}, \ldots, v_{n}\right)$ from left to right, and computes a minimum 1PC of the graph $G\left(\left[S \cup\left\{v_{i}\right\}\right]\right)$ from a minimum 1PC of $G([S]), 1 \leq i \leq n$, where $S=\left\{v_{1}, v_{2}, \ldots, v_{i-1}\right\}$, using the operations connect, insert, bridge, new_path, and connect_break. It is easy to see that each one of these operations takes time linear to the size of the graph $G\left[\left\{v_{1}, v_{2}, \ldots, v_{i-1}\right\}\right]$. Note that, if no vertex is specified to be terminal the algorithm uses only the operations connect, bridge or new_path and, thus, it constructs a minimum PC $\mathcal{P}(G)$ of $G$ in $O\left(n^{2}\right)$ time.

Let $G$ be an interval graph on $n$ vertices and $m$ edges and let $\mathcal{T}$ be a terminal set containing a vertex $v \in V(G)$. Suppose that Algorithm 1PC_INTERVAL processes vertex $v_{i}, 1 \leq i \leq n$, and $t$ is the index of the terminal vertex; we consider the following cases:

Case $i<t$. The algorithm processes vertex $v_{i}$, where $i<t$. In this case, one of the following operations is performed: connect, bridge or new_path. Each operation includes searching for endpoints of paths in $\mathcal{P}_{\mathcal{T}}\left(G\left[\left\{v_{1}, v_{2}, \ldots, v_{i-1}\right\}\right]\right)$ that are neighbors of $v_{i}$. Thus, a minimum $1 \mathrm{PC}$ of $G\left[\left\{v_{1}, v_{2}, \ldots, v_{i}\right\}\right]$ is constructed in time linear to the size of $G\left[\left\{v_{1}, v_{2}, \ldots, v_{i-1}\right\}\right]$.

Case $i=t$. The algorithm processes vertex $v_{t}$. Then, either operation connect or operation new_path is performed; specifically, the procedure process_ terminal is searching for the leftmost endpoint, say, $v_{\ell}$, of a path in $\mathcal{P}_{\mathcal{T}}\left(G\left[\left\{v_{1}, v_{2}\right.\right.\right.$, $\left.\left.\ldots, v_{t-1}\right\}\right]$ ) being a neighbor of $v_{t}$, and, either connects $v_{t}$ to $v_{\ell}$ or creates a new trivial path. Thus, a minimum $1 \mathrm{PC}$ of $G\left[\left\{v_{1}, v_{2}, \ldots, v_{t}\right\}\right]$ is constructed in time linear to the size of $G\left[\left\{v_{1}, v_{2}, \ldots, v_{t-1}\right\}\right]$.

Case $i>t$. The algorithm processes vertex $v_{i}$, where $t<i$. Unless the computation of a minimum PC of $G\left[\left\{v_{1}, v_{2}, \ldots, v_{i-1}\right\}-\left\{v_{t}\right\}\right]$ is required or a minimum 1PC of $G\left[\left\{v_{1}, \ldots, v_{t}, \ldots, v_{i-1}\right\}\right]$ is reconstructed, the algorithm processes vertex $v_{i}$ using the operations connect, insert, bridge, new_path, and connect_break. Since each one of these operations takes $O(i)$ time, in this case a minimum 1PC of $G\left[\left\{v_{1}, \ldots, v_{t}, \ldots, v_{i}\right\}\right]$ is computed in $O(i)$ time. In the case where a minimum PC of $G\left[\left\{v_{1}, v_{2}, \ldots, v_{i-1}\right\}-\left\{v_{t}\right\}\right]$ is computed or a minimum 1PC of $G\left[\left\{v_{1}, \ldots, v_{t}, \ldots, v_{i-1}\right\}\right]$ is reconstructed, the algorithm computes a minimum 1PC of $G\left[\left\{v_{1}, v_{2}, \ldots, v_{i}\right\}\right]$ in $O\left(i^{2}\right)$ time. Thus, a minimum $1 \mathrm{PC}$ of $G\left[\left\{v_{1}, v_{2}, \ldots, v_{i}\right\}\right]$ is constructed in time quadratic to the size of $G\left[\left\{v_{1}, v_{2}, \ldots, v_{i-1}\right\}\right]$.

Taking into consideration the time complexity of the operations performed by the algorithm in each case, we conclude that it computes a minimum $1 \mathrm{PC} \mathcal{P}_{\mathcal{T}}(G)$ of $G$ 
in $O\left(n^{3}\right)$ time and, since no additional space is needed, requires linear space. Recall that the ordering $\pi$ of the vertices is constructed in linear time [24]. Hence, we can state the following result.

Theorem 4.2 Let $G$ be an interval graph on $n$ vertices and let $\mathcal{T}$ be a subset of $V(G)$ containing a single vertex. A minimum 1-fixed-endpoint path cover of $G$ with respect to $\mathcal{T}$ can be computed in $O\left(n^{3}\right)$ time.

\section{Related Results on Convex and Biconvex Graphs}

Based on the results for the 1PC problem on interval graphs, and also on the reduction described by Müller in [20], we study the HP and 1HP problems on convex and biconvex graphs. A bipartite graph $G(X, Y ; E)$ is called $X$-convex (or simply convex) if there exists an ordering of its vertices such that for all $y \in Y$ the vertices of $N(y)$ are consecutive [20]; $G$ is biconvex if it is convex on both $X$ and $Y$.

In this section, we solve the HP and 1HP problems on a biconvex graph $G(X, Y ; E)$. Moreover, we show that the HP problem on an $X$-convex graph $G(X, Y ; E)$ on $n$ vertices can be solved in $O\left(n^{4}\right)$ time if $|X|=|Y|$ or $|X|-|Y|=1$ and a $1 \mathrm{HP}$ starting at vertex $u$, if there exists, can be computed in $O\left(n^{3}\right)$ time if $(|X|=|Y|$ and $u \in Y)$ or $|X|-|Y|=1$.

We next describe an algorithm for the HP problem on a biconvex graph $G(X, Y ; E)$. To simplify our description we use two functions, namely Minimum _HP $(G)$ and Minimum_1PC $(G, u)$ : the function Minimum_HP $(G)$ corresponds to the algorithm for computing a minimum path cover of an interval graph $G$ described in [2], while Minimum_1PC $(G, u)$ corresponds to the algorithm for computing a minimum path cover with specified endpoint $u$ of an interval graph $G$ described in this paper.

\section{Algorithm HP_BICONVEX}

Input: a biconvex graph $G(X, Y ; E)$ on $n$ vertices;

Output: a Hamiltonian path of $G$, if one exists:

1. if ||$X|-| Y||>1$ then return $G$ does not have a Hamiltonian path;

2. if $|X|=|Y|$ then

construct the interval graph $G^{\prime}$ such that:

$V\left(G^{\prime}\right)=X \cup Y, E\left(G^{\prime}\right)=E \cup E_{Y}$, where $E_{Y}$ is as follows:

$y_{1} y_{2} \in E_{Y}$ iff $y_{1}, y_{2} \in Y$ and $N\left(y_{1}\right) \cap N\left(y_{2}\right) \neq \emptyset$;

if there exists $y_{j} \in Y$ such that $\left|N\left(y_{j}\right)\right|=1$ then

$\mathcal{P}_{\mathcal{T}}(G) \leftarrow$ Minimum_1PC $\left(G^{\prime}, y_{j}\right)$;

if $\lambda_{\mathcal{T}}(G)=1$ then return $\mathcal{P}_{\mathcal{T}}(G)$

else return $G$ does not have a Hamiltonian path;

else

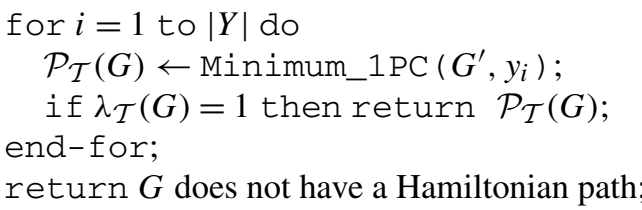


3. if $|X|-|Y|=1$ then

$\mathcal{P}_{\mathcal{T}}(G) \leftarrow$ Minimum_HP $\left(G^{\prime}\right)$;

if $\lambda_{\mathcal{T}}(G)=1$ then return $\mathcal{P}_{\mathcal{T}}(G)$;

else return $G$ does not have a Hamiltonian path;

4. if $|Y|-|X|=1$ then

construct the interval graph $G^{\prime}$ such that:

$V\left(G^{\prime}\right)=X \cup Y, E\left(G^{\prime}\right)=E \cup E_{X}$, where $E_{X}$ is as follows:

$x_{1} x_{2} \in E_{X}$ iff $x_{1}, x_{2} \in X$ and $N\left(x_{1}\right) \cap N\left(x_{2}\right) \neq \emptyset$;

$\mathcal{P}_{\mathcal{T}}(G) \leftarrow$ Minimum_HP $\left(G^{\prime}\right)$;

if $\lambda_{\mathcal{T}}(G)=1$ then return $\mathcal{P}_{\mathcal{T}}(G)$;

else return $G$ does not have a Hamiltonian path;

End_of_Algorithm HP_BICONVEX.

Observation 5.1 Uehara and Uno in [31] claim that the HP problem on a biconvex graph $G(X, Y ; E)$ on $n$ vertices can be solved in $O\left(n^{2}\right)$ time even if $|X|=|Y|$. Specifically, they claim that $G$ has an HP iff the interval graph $G^{\prime}$ has an HP, where $G^{\prime}$ is an interval graph such that $V\left(G^{\prime}\right)=X \cup Y$ and $E\left(G^{\prime}\right)=E \cup E_{Y}$, where $E_{Y}$ is as follows: $y_{1} y_{2} \in E_{Y}$ iff $y_{1}, y_{2} \in Y$ and $N\left(y_{1}\right) \cap N\left(y_{2}\right) \neq \emptyset$. However, this is not true, since there exists a counterexample, which is presented in Fig. 7. Indeed, the biconvex graph $G$ of Fig. 7 does not have an HP while for $G^{\prime}$ we have $P=\left(x_{1}, y_{2}, x_{2}, y_{1}, y_{4}, x_{3}, y_{3}, x_{4}\right)$. Suppose that we construct an interval graph $G^{\prime}$ such that $V\left(G^{\prime}\right)=X \cup Y$ and $E\left(G^{\prime}\right)=E \cup E_{X}$, where $E_{X}$ is as follows: $x_{1} x_{2} \in E_{X}$ iff $x_{1}, x_{2} \in X$ and $N\left(x_{1}\right) \cap N\left(x_{2}\right) \neq \emptyset$. Then, $G^{\prime}$ has an HP, that is, $P=\left(y_{4}, x_{3}, y_{3}, x_{4}, x_{1}, y_{2}, x_{2}, y_{1}\right)$. Thus, there exists no algorithm with time complexity $O\left(n^{2}\right)$ and we can state the following result.

Theorem 5.1 The Hamiltonian path problem on a biconvex graph $G$ on $n$ vertices can be solved in $O\left(n^{4}\right)$ time.

Similarly, we show that the Hamiltonian path problem on an $X$-convex graph $G(X, Y ; E)$ on $n$ vertices can be solved in $O\left(n^{4}\right)$ time when $|X|=|Y|$ or $|X|-|Y|=$ 1. It is easy to see that if $|Y|-|X|=1$ then the $X$-convex graph $G(X, Y ; E)$ has a Hamiltonian path iff the interval graph $G^{\prime}$ has a 2 HP between any two vertices of $Y$. Thus, we can state the following result.

Corollary 5.1 The Hamiltonian path problem on an $X$-convex graph $G(X, Y ; E)$ on $n$ vertices can be solved in $O\left(n^{4}\right)$ time if $|X|=|Y|$ or $|X|-|Y|=1$.

Fig. 7 A biconvex graph $G=(X, Y ; E)$ with $|X|=|Y|$

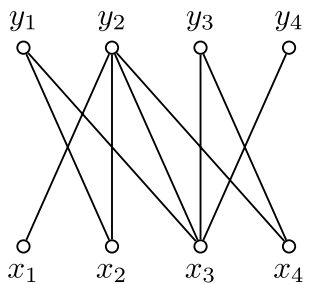


We next describe an algorithm for the 1HP problem on a biconvex graph $G(X, Y ; E)$. Recall that the function Minimum_1PC $(G, u)$ corresponds to the algorithm for computing a minimum path cover with specified endpoint $u$ of an interval graph $G$ described in this paper.

\section{Algorithm 1HP_BICONVEX}

Input: a biconvex graph $G(X, Y ; E)$ on $n$ vertices and a vertex $y_{t} \in Y$;

Output: a Hamiltonian path of $G$ starting at vertex $y_{t}$, if one exists:

1. if ||$X|-| Y||>1$ then return $G$ does not have a $1 \mathrm{HP}$;

2. if $|X|=|Y|$ then

construct the interval graph $G^{\prime}$ such that:

$V\left(G^{\prime}\right)=X \cup Y, E\left(G^{\prime}\right)=E \cup E_{Y}$, where $E_{Y}$ is as follows:

$y_{1} y_{2} \in E_{Y}$ iff $y_{1}, y_{2} \in Y$ and $N\left(y_{1}\right) \cap N\left(y_{2}\right) \neq \emptyset$;

$\mathcal{P}_{\mathcal{T}}(G) \leftarrow$ Minimum_1PC $\left(G^{\prime}, y_{t}\right)$;

if $\lambda_{\mathcal{T}}(G)=1$ then return $\mathcal{P}_{\mathcal{T}}(G)$;

else return $G$ does not have a $1 \mathrm{HP}$;

3. if $|X|-|Y|=1$ then return $G$ does not have a 1HP;

4. if $|Y|-|X|=1$ then

construct the interval graph $G^{\prime}$ such that:

$V\left(G^{\prime}\right)=X \cup Y, E\left(G^{\prime}\right)=E \cup E_{X}$, where $E_{X}$ is as follows:

$x_{1} x_{2} \in E_{X}$ iff $x_{1}, x_{2} \in X$ and $N\left(x_{1}\right) \cap N\left(x_{2}\right) \neq \emptyset$;

$\mathcal{P}_{\mathcal{T}}(G) \leftarrow$ Minimum_1PC $\left(G^{\prime}, y_{t}\right)$;

if $\lambda_{\mathcal{T}}(G)=1$ then return $\mathcal{P}_{\mathcal{T}}(G)$;

else return $G$ does not have a $1 \mathrm{HP}$;

End_of_Algorithm 1HP_BICONVEX.

Since the function Minimum_1PC requires $O\left(n^{3}\right)$ time to compute a $1 \mathrm{HP}$ of an interval graph on $n$ vertices and the graph $G^{\prime}$ can be constructed in $O\left(|X \cup Y|^{2}\right)$ time [20], Algorithm 1HP_BICONVEX returns a 1HP, if there exists, of a biconvex graph on $n$ vertices in $O\left(n^{3}\right)$ time. Hence, we can state the following result.

Theorem 5.2 Let $G$ be a biconvex graph on $n$ vertices and let $u$ be a vertex of $V(G)$. The 1HP problem on $G$ and $u$ can be solved in $O\left(n^{3}\right)$ time.

Let $G(X, Y ; E)$ be an $X$-convex graph on $n$ vertices and let $u$ be a vertex of $V(G)$. Similarly, we show that the 1HP problem on $G$ and $u$ can be solved in $O\left(n^{3}\right)$ time when $(|X|=|Y|$ and $u \in Y)$ or $|X|-|Y|=1$. Clearly, if $|Y|-|X|=1$ and $u \in X$ then $G$ does not have a 1HP. It is easy to see that if $(|Y|-|X|=1$ and $u \in Y)$ or $(|X|=|Y|$ and $u \in X)$ then the $X$-convex graph $G(X, Y ; E)$ has a Hamiltonian path if the interval graph $G^{\prime}$ has a 2 HP between $u$ and a vertex of $Y$. Thus, we can state the following result.

Corollary 5.2 Let $G(X, Y ; E)$ be an $X$-convex graph on $n$ vertices and let $u$ be a vertex of $V(G)$. The $1 \mathrm{HP}$ problem on $G$ and $u$ can be solved in $O\left(n^{3}\right)$ time if $(|X|=|Y|$ and $u \in Y)$ or $|X|-|Y|=1$. If $|Y|-|X|=1$ and $u \in X$ then $G$ does not have a $1 \mathrm{HP}$. 


\section{Concluding Remarks}

In this paper we presented a polynomial-time algorithm for the 1PC problem on interval graphs and, thus, we answered the question left open by Damaschke [8] of whether the 1HP problem is polynomially solvable on interval graphs. We also presented polynomial-time algorithms for the HP and 1HP problems on biconvex graphs, and showed that the HP problem on $X$-convex graphs $G(X, Y, E)$ with $|Y|-|X|=1$, which was left open in [31], has polynomial solution iff the $2 \mathrm{HP}$ problem on interval graphs is polynomial.

An interesting open question is whether the $k$-fixed-endpoint path cover problem $(k \mathrm{PC})$ can be polynomially solved on interval graphs. Note that, the $k \mathrm{PC}$ problem generalizes both the $1 \mathrm{HP}$ and $2 \mathrm{HP}$ problems; the complexity status of the $2 \mathrm{HP}$ problem on interval graphs remains an open question.

\section{References}

1. Adhar, G.S., Peng, S.: Parallel algorithm for path covering, Hamiltonian path, and Hamiltonian cycle in cographs. In: International Conference on Parallel Processing, vol. III: Algorithms and Architecture, pp. 364-365. Pennsylvania State University Press, Pennsylvania (1990)

2. Arikati, S.R., Rangan, C.P.: Linear algorithm for optimal path cover problem on interval graphs. Inf. Process. Lett. 35, 149-153 (1990)

3. Asdre, K., Nikolopoulos, S.D.: A linear-time algorithm for the $k$-fixed-endpoint path cover problem on cographs. Networks 50, 231-240 (2007)

4. Asdre, K., Nikolopoulos, S.D.: A polynomial solution for the $k$-fixed-endpoint path cover problem on proper interval graphs. In: Proc. of the 18th International Conference on Combinatorial Algorithms (IWOCA'07), Lake Macquarie, Newcastle, Australia (2007)

5. Bertossi, A.A.: Finding Hamiltonian circuits in proper interval graphs. Inf. Process. Lett. 17, 97-101 (1983)

6. Bertossi, A.A., Bonuccelli, M.A.: Finding Hamiltonian circuits in interval graph generalizations. Inf. Process. Lett. 23, 195-200 (1986)

7. Brandstädt, A., Le, V.B., Spinrad, J.: Graph Classes-A Survey. SIAM Monographs in Discrete Mathematics and Applications. SIAM, Philadelphia (1999)

8. Damaschke, P.: Paths in interval graphs and circular arc graphs. Discrete Math. 112, $49-64$ (1993)

9. Fortune, S., Hopcroft, J.E., Wyllie, J.: The directed subgraph homeomorphism problem. J. Theor. Comput. Sci. 10, 111-121 (1980)

10. Garey, M.R., Johnson, D.S.: Computers and Intractability: A Guide to the Theory of NP-Completeness. Freeman, San Francisco (1979)

11. Garey, M.R., Johnson, D.S., Tarjan, R.E.: The planar Hamiltonian circuit problem is NP-complete. SIAM J. Comput. 5, 704-714 (1976)

12. Golumbic, M.C.: Algorithmic Graph Theory and Perfect Graphs. Academic Press, New York (1980) (2nd edn., in Ann. Discrete Math. 57, Elsevier, 2004)

13. Hsieh, S.Y.: An efficient parallel strategy for the two-fixed-endpoint Hamiltonian path problem on distance-hereditary graphs. J. Parallel Distributed Comput. 64, 662-685 (2004)

14. Hsieh, S.Y., Ho, C.W., Hsu, T.S., Ko, M.T.: The Hamiltonian problem on distance-hereditary graphs. Discrete Appl. Math. 154, 508-524 (2006)

15. Hung, R.W., Chang, M.S.: Linear-time algorithms for the Hamiltonian problems on distancehereditary graphs. Theor. Comput. Sci. 341, 411-440 (2005)

16. Hung, R.W., Chang, M.S.: Solving the path cover problem on circular-arc graphs by using an approximation algorithm. Discrete Appl. Math. 154, 76-105 (2006)

17. Karp, R.M.: On the complexity of combinatorial problems. Networks 5, 45-68 (1975)

18. Keil, J.M.: Finding Hamiltonian circuits in interval graphs. Inf. Process. Lett. 20, 201-206 (1985)

19. Lin, R., Olariu, S., Pruesse, G.: An optimal path cover algorithm for cographs. Comput. Math. Appl. 30, 75-83 (1995) 
20. Müller, H.: Hamiltonian circuits in chordal bipartite graphs. Discrete Math. 156, 291-298 (1996)

21. Nakano, K., Olariu, S., Zomaya, A.Y.: A time-optimal solution for the path cover problem on cographs. Theor. Comput. Sci. 290, 1541-1556 (2003)

22. Nikolopoulos, S.D.: Parallel algorithms for Hamiltonian problems on quasi-threshold graphs. J. Parallel Distributed Comput. 64, 48-67 (2004)

23. Park, J.H.: One-to-many disjoint path covers in a graph with faulty elements. In: Proc. of the 10th International Computing and Combinatorics Conference (COCOON'04), pp. 392-401 (2004)

24. Ramalingam, G., Rangan, C.P.: A unified approach to domination problems on interval graphs. Inf. Process. Lett. 27, 271-274 (1988)

25. Robertson, N., Seymour, P.D.: Graph minors. XIII. The disjoint paths problem. J. Comb. Theory Ser. B 63, 65-110 (1995)

26. Seymour, P.D.: Disjoint paths in graphs. Discrete Math. 29, 293-309 (1980)

27. Shiloach, Y.: A polynomial solution to the undirected two paths problem. J. Assoc. Comput. Mach. 27, 445-456 (1980)

28. Srikant, R., Sundaram, R., Singh, K.S., Rangan, C.P.: Optimal path cover problem on block graphs and bipartite permutation graphs. Theor. Comput. Sci. 115, 351-357 (1993)

29. Suzuki, Y., Kaneko, K., Nakamori, M.: Node-disjoint paths algorithm in a transposition graph. IEICE Trans. Inf. Syst. E89-D, 2600-2605 (2006)

30. Thomassen, C.: 2-linked graphs. Eur. J. Comb. 1, 371-378 (1980)

31. Uehara, R., Uno, Y.: On computing longest paths in small graph classes. Int. J. Found. Comput. Sci. 18, 911-930 (2007) 